ARTICLE

\title{
FAM111A protects replication forks from protein obstacles via its trypsin-like domain
}

\author{
Yusuke Kojima1', Yuka Machida1, Sowmiya Palani², Thomas R. Caulfield (1D ${ }^{3}$, Evette S. Radisky4,
} Scott H. Kaufmann (10 ${ }^{1,5}$ \& Yuichi J. Machida ${ }^{1,5}{ }^{\circ}$

Persistent protein obstacles on genomic DNA, such as DNA-protein crosslinks (DPCs) and tight nucleoprotein complexes, can block replication forks. DPCs can be removed by the proteolytic activities of the metalloprotease SPRTN or the proteasome in a replicationcoupled manner; however, additional proteolytic mechanisms may exist to cope with the diversity of protein obstacles. Here, we show that FAM111A, a PCNA-interacting protein, plays an important role in mitigating the effect of protein obstacles on replication forks. This function of FAM111A requires an intact trypsin-like protease domain, the PCNA interaction, and the DNA-binding domain that is necessary for protease activity in vivo. FAM111A, but not SPRTN, protects replication forks from stalling at poly(ADP-ribose) polymerase 1 (PARP1)DNA complexes trapped by PARP inhibitors, thereby promoting cell survival after drug treatment. Altogether, our findings reveal a role of FAM111A in overcoming protein obstacles to replication forks, shedding light on cellular responses to anti-cancer therapies.

\footnotetext{
${ }^{1}$ Department of Oncology, Mayo Clinic, Rochester, MN 55905, USA. ${ }^{2}$ Department of Biochemistry and Molecular Biology, Mayo Clinic, Rochester, MN 55905, USA. ${ }^{3}$ Department of Neuroscience, Mayo Clinic, Jacksonville, FL 32224, USA. ${ }^{4}$ Department of Cancer Biology, Mayo Clinic, Jacksonville, FL 32224 USA. ${ }^{5}$ Department of Molecular Pharmacology and Experimental Therapeutics, Mayo Clinic, Rochester, MN 55905, USA. ${ }^{凶}$ email: machida.yuichi@mayo.edu
} 
C ollision of the DNA replication machinery with replication fork obstacles causes replication fork stalling and doublestrand DNA breaks (DSBs), posing a great threat to genome integrity ${ }^{1-3}$. In particular, protein obstacles such as DNAprotein crosslinks (DPCs) have deleterious effects on replication forks due to their bulky nature. DPCs can form by various mechanisms, with differing sizes and DNA-crosslink structures ${ }^{4-6}$. For example, crosslinking chemicals, such as formaldehyde and reactive oxygen species, covalently link proteins to DNA nonspecifically. In contrast, abortive enzymatic reactions can produce specific DPCs as represented by topoisomerase 1 cleavage complexes (TOP1ccs), which result from trapping of TOP1 covalently bound to DNA in an intermediate step of the enzyme reaction. Because of the severe consequences of blocking replication forks, induction of DPCs or nucleoprotein complexes is a common mechanism for anti-cancer drugs. TOP1 inhibitors, such as topotecan and its prototype camptothecin (CPT), poison TOP1, thereby inducing cytotoxic levels of TOP $1 \mathrm{ccs}^{7}$. On the other hand, poly(ADP-ribose) polymerase (PARP) inhibitors (PARPis) induce nucleoprotein complexes by trapping PARP1 protein at DNA single-strand breaks (SSBs) during the SSB repair process ${ }^{8-10}$. Through strong DNA binding that mimics a DPC, PARP1-DNA complexes block DNA replication and cause DSBs ${ }^{11-13}$. Since blocked replication forks and DSBs require the homologous recombination (HR) pathway for repair, PARPis are particularly toxic to HR-deficient tumors with BRCA1/2 mutations ${ }^{14,15}$.

The discovery of the metalloproteases, Wss 1 in yeasts and SPRTN in higher eukaryotes, revealed a previously unrecognized contribution of proteolysis to DPC repair ${ }^{16-25}$. The SprT metalloprotease domain of SPRTN contains a $\mathrm{Zn}^{2+}$-binding subdomain that binds single-strand DNA (ssDNA), which activates SPRTN $^{26}$. SPRTN localizes to replication forks ${ }^{20,27}$ and degrades DPC proteins upon replication fork collisions ${ }^{28,29}$. SPRTN deficiency causes progeria and an increased incidence of liver cancer in humans and mice, highlighting the importance of DPC repair $^{27,30-32}$. In addition to SPRTN, DPCs can also be resolved using the proteasome, which can degrade DPCs following replication-coupled polyubiquitination of DPC proteins ${ }^{29}$. Furthermore, replication-independent sumoylation of DPC proteins has also been reported 29,33 and ACRC/GCNA family SprT proteases are involved in DPC repair in germline cells ${ }^{33}$. On the other hand, it is unknown whether these DPC repair mechanisms play a role in preventing replication fork stalling at noncovalently linked protein-DNA complexes. Indeed, we previously reported that Sprtn hypomorphic mouse embryonic fibroblasts (MEFs) are not hypersensitive to PARPis, implying that SPRTN is unlikely to be involved in removal of PARP1-DNA complexes $^{27}$. Therefore, it is currently unknown whether a cellular mechanism exists to remove trapped PARPs, but such a system would be expected to undermine the therapeutic effect of PARPis.

In this study, we demonstrate that the putative serine protease FAM111A (Family with sequence similarity 111 member A) protects replication forks from stalling at PARP1-DNA complexes and TOP1ccs. FAM111A was originally identified as a replication fork protein by nascent chromatin capture proteomics and shown to interact with PCNA through its PCNA-interacting peptide (PIP) box ${ }^{34}$. Although it was proposed that FAM111A plays a role in PCNA loading, its function at ongoing replication forks was unknown. The presence of a trypsin-like protease domain in FAM111A and its localization to replication forks prompted us to investigate whether FAM111A is involved in DPC removal during DNA replication. Our findings uncover a critical role of FAM111A in promoting DNA replication fork progression not only at DPCs, but also at PARP1-DNA nucleoprotein complexes.

\section{Results}

FAM111A KO cells are sensitive to PARP and TOP1 inhibitors. To investigate whether FAM111A is important for mitigating the effect of protein obstacles to replication forks, we examined whether FAM111A depletion affects sensitivities to a PARPi and a TOP1 poison. FAM111A-knockout (KO) by CRISPR/Cas9 (Fig. 1a, Supplementary Fig. 1, and Supplementary Table 1) rendered cells hypersensitive to the PARPi niraparib, which exhibits strong PARP-trapping effect ${ }^{9}$, and the TOP1 inhibitor CPT (Fig. 1b). Similar results were also obtained with additional FAM111A KO clones obtained using CRISPR/Cas9 targeting different sequences of the gene (Supplementary Figs. 1, 2a, b and Supplementary Table 1). Furthermore, FAM111A KO cells were sensitive to another PARPi with a strong PARP-trapping effect ${ }^{10}$, talazoparib (Supplementary Fig. 2c). In addition, FAM111A KO cells showed mild sensitization to etoposide (a topoisomerase II inhibitor that covalently traps TOP2), 5-aza-dC (a DNMT inhibitor that traps DNMT isoenzymes on DNA), and formaldehyde (Supplementary Fig. 2d). On the other hand, FAM111A KO cells were not sensitized to ionizing radiation (IR) or cisplatin (a DNA crosslinker) (Fig. 1b and Supplementary Fig. 2d).

The hypersensitivity of FAM111A KO cells to niraparib was also associated with a marked increase in apoptotic cell death as shown by Annexin V staining (Fig. 1c, d). Activation of the HR pathway was intact in FAM111A KO cells when they were treated with PARPi as shown by RAD51 focus formation (Supplementary Fig. 2e, f), indicating that an HR defect does not account for the PARPi hypersensitivity of FAM111A KO cells. This conclusion is also supported by the fact that FAM111A KO cells were not sensitive to IR (Fig. 1b), which relies on an intact HR pathway for optimal repair. Taken together, these results suggest that FAM111A protects cells from several anti-cancer drugs, especially PARP and TOP1 inhibitors.

FAM111A directly binds to ssDNA through its central region. Because FAM111A localizes to replication forks ${ }^{34}$, we hypothesized that FAM111A might have DNA-binding activity. We tested this possibility by electrophoretic mobility shift assays (EMSA) using purified full-length FAM111A protein (Supplementary Fig. 3a) and various forms of labeled DNA oligonucleotides, including ssDNA, double-strand (ds) DNA, Y fork, and dsY fork (Supplementary Fig. 3b). FAM111A displayed robust binding to ssDNA and Y-fork DNA, weaker binding to dsDNA, and undetectable binding to dsY fork DNA (Fig. 2a). These results suggest that FAM111A exhibits preferential binding to ssDNA, as ssDNA structures were common between the ssDNA and Y fork DNA oligos used in these experiments. Supershift assays using anti-FAM111A antibody verified that the mobility shift was due to FAM111A rather than possible contaminants in the protein preparation (Fig. 2b). Binding of FAM111A to ssDNA was also confirmed using fluorescence polarization assays, which showed an equilibrium dissociation constant $\left(\mathrm{K}_{\mathrm{d}}\right)$ of $\sim 60 \mathrm{nM}$ for ssDNA binding (Fig. 2c).

We next sought to map the DNA-binding domain of FAM111A. EMSA using various fragments of FAM111A (Fig. 2d and Supplementary Fig. 3c) revealed that the central region (176-282) was sufficient to bind to ssDNA (Fig. 2e). On the other hand, the ssDNA-binding activity of the N-terminal FAM111A fragment (1-282) was diminished with varying degrees when a part of the central region was deleted (Supplementary Fig. 3d-f). These results suggested that the central region (176-282) is necessary and sufficient for the ssDNA binding of FAM111A.

To identify amino acid residues in the FAM111A central region that are crucial for ssDNA binding, we introduced point mutations in aromatic or positively charged amino acid residues 


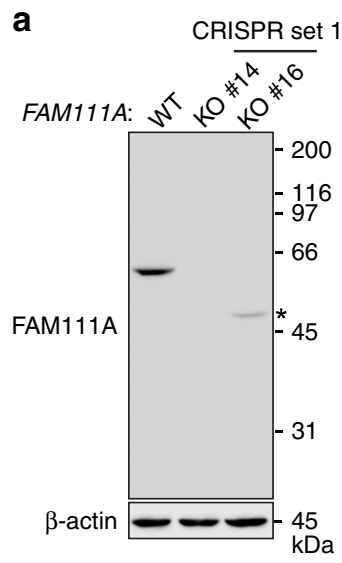

C

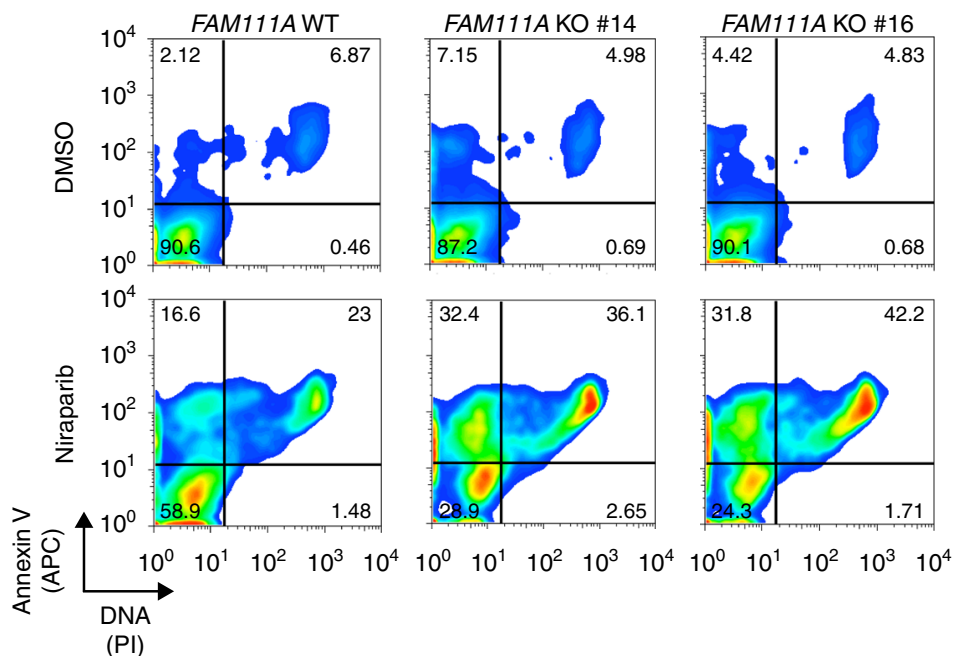

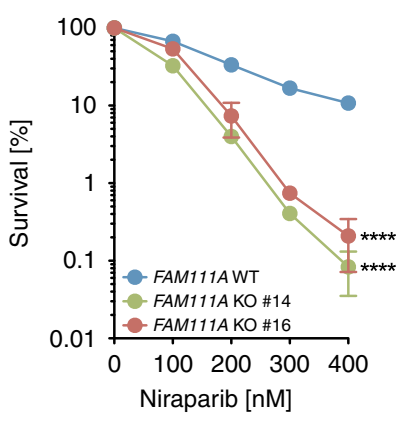
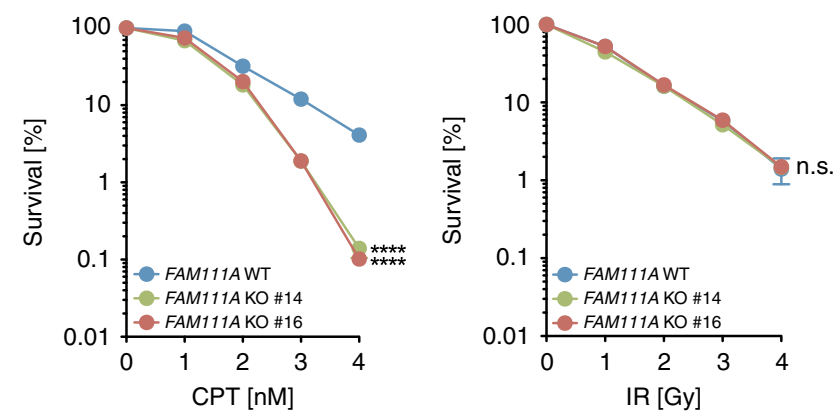

d

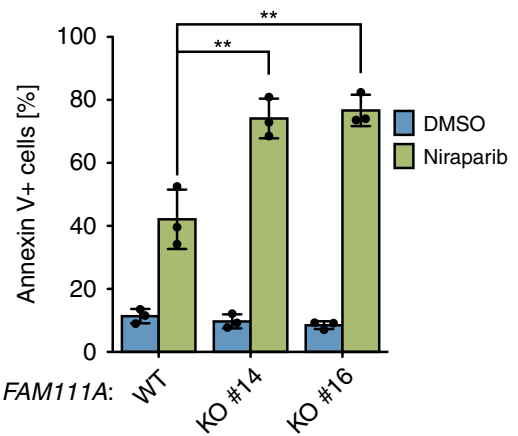

Fig. 1 FAM111A deficiency sensitizes cells to PARPis and CPT. a Knockout of FAM111A by CRISPR/Cas9 (set 1 gRNA). Parental HAP1 (WT) and FAM111A $\mathrm{KO}$ clones (KO \#14 and \#16) were analyzed for the indicated proteins by Western blotting. ${ }^{\star}$, truncated FAM111A protein. $\mathbf{b}$ Clonogenic survival assays. Cells analyzed in a were cultured in the presence of the indicated concentration of niraparib or CPT for 6 days. For ionizing radiation (IR), cells were irradiated at the indicated doses and cultured for 6 days. Results shown are representative of three independent experiments and values are mean \pm s.d. of technical replicates $(n=3)$. $p$ values were calculated relative to FAM111A WT. ${ }^{\star \star \star \star} p<0.0001$; n.s. not significant (two-tailed unpaired $t$-test). c Analyses of cell death by Annexin V/PI staining. After treatment with $1 \mu \mathrm{M}$ niraparib or DMSO for $48 \mathrm{~h}$, cells stained with Annexin V-APC and PI were analyzed by flow cytometry. Results shown are representative of three independent experiments and percentages of cells in each quadrant are indicated. $\mathbf{d}$ Quantification of Annexin V-positive cells. Experiments were performed as in c. Values are mean \pm s.d. of independent experiments $(n=3) .{ }^{\star \star} p<0.01$ (two-tailed unpaired $t$-test). Source data are provided as a Source Data file.

based on a previous report showing that those amino acid residues are involved in ssDNA binding ${ }^{35}$. We particularly focused on amino acids conserved among species or stretches of positively charged amino acids (Supplementary Fig. 3g). EMSA using MBP-FAM111A 1-282 with point mutations revealed that the F231A mutation had the largest effect on ssDNA binding among the mutants tested (Supplementary Fig. 3h, i). The F231A mutation decreased DNA binding to both ssDNA and Y-fork substrates (Fig. 2f), and similar results were obtained with the minimum DNA-binding domain (176-282) (Supplementary Fig. 3j-1). All of these data suggest that Phe231 plays a key role in FAM111A binding to ssDNA.

Disease-causing mutations enhance FAM111 A cleavage in vivo. FAM111A contains a trypsin-like domain at its C-terminus with an intact catalytic triad consisting of His385, Aps439 and Ser541, a characteristic feature of the $\mathrm{S} 1$ serine protease family ${ }^{36,37}$. Given that proteases often exhibit autocleavage activity, we set out to find processed fragments of FAM111A in vivo to study the putative protease activity. When exogenous FAM111A was expressed in 293T cells, which lack endogenous FAM111A expression for an unknown reason (Supplementary Fig. 4a), a small FAM111A fragment $(\sim 40 \mathrm{kDa})$, as well as full-length protein, was detected on the immunoblot using the anti-FAM111A antibody that recognizes the $\mathrm{N}$-terminal region (Fig. 3a). This fragment was not observed with the mutant that contains a substitution of the active site serine to an alanine (S541A). These results raised the possibility that FAM111A might have autocleavage activity in vivo. Interestingly, germline mutations have been found in FAM111A in patients with Kenny-Caffey syndrome (KCS) and osteocraniostenosis (OCS) ${ }^{38-41}$, and it has been postulated that these mutations may cause gain-of-function FAM111A activity because the mutations are always heterozygous and no loss-of-function mutations (i.e., deletions and truncations) have been reported ${ }^{38}$. We therefore tested whether the mutations found in KCS and OCS patients produce constitutively active FAM111A. For all the patient mutants tested (R569H and Y511H for KCS; S342del and D528G for OCS), the levels of full-length protein were reduced concomitant with a 
a
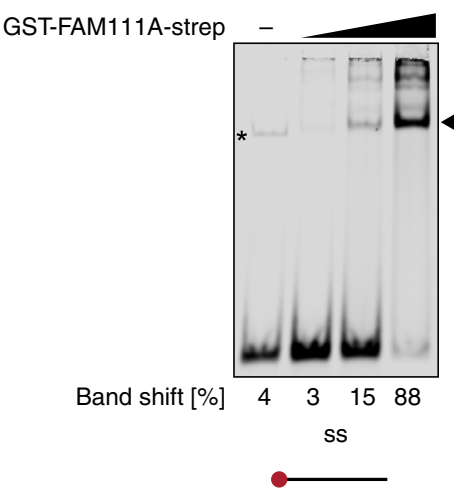

C

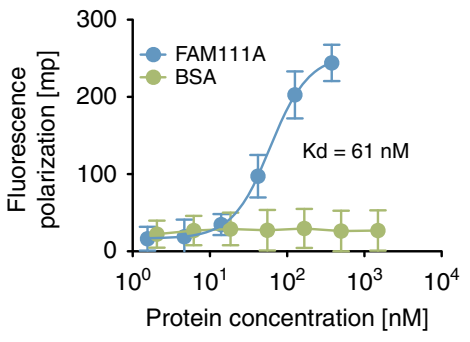

b

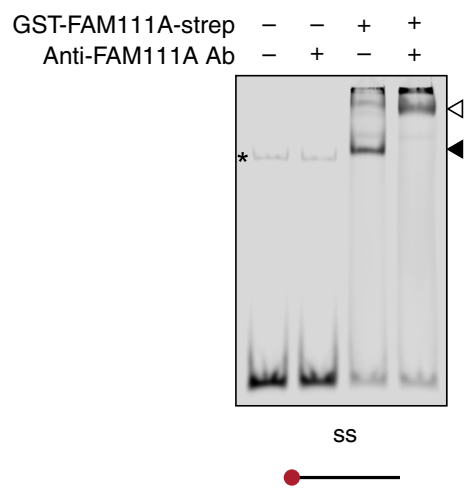

ds $Y$ fork

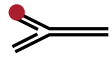

d

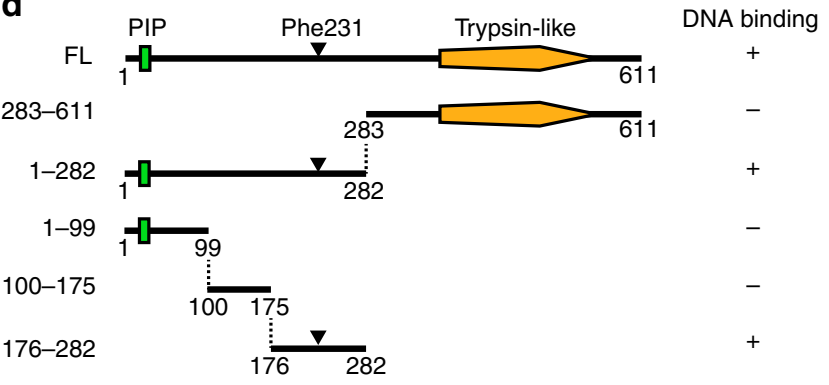

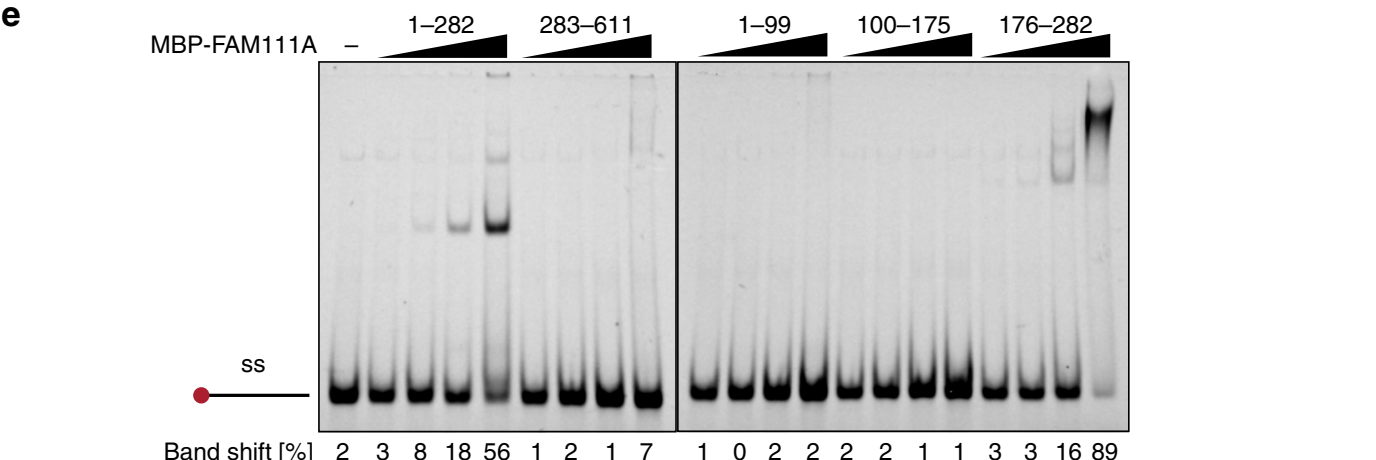

Band shift [\%] $2 \begin{array}{lllllllllllllllllllll}2 & 3 & 8 & 18 & 56 & 1 & 2 & 1 & 7 & 1 & 0 & 2 & 2 & 2 & 2 & 1 & 1 & 3 & 3 & 16 & 89\end{array}$

f

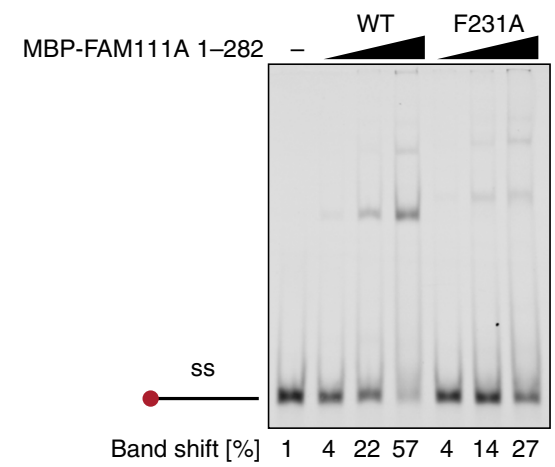

large increase in the levels of the processed fragment (Fig. 3b, lanes 4, 6, 8, and 10). Addition of an active site mutation (Ser541, S541A) to these patient mutants restored full-length protein expression and eliminated the processed fragment (Fig. 3b, lanes $5,7,9$, and 11). These results suggest that the FAM111A patient mutants are constitutively active forms that result in the conversion of the full-length protein to smaller fragments through a possible autocleavage mechanism.

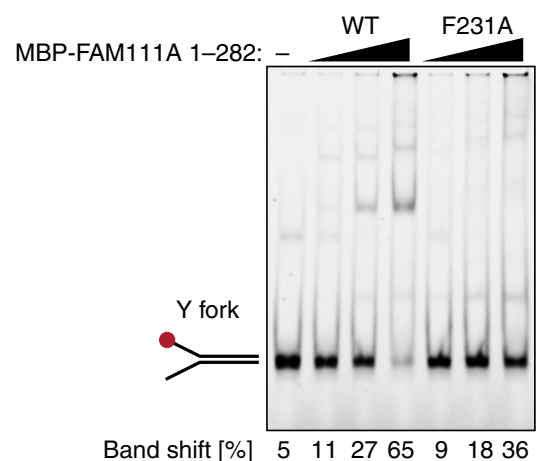

FAM111A activity exhibits chymotrypsin-like specificity. The S1 family serine proteases exhibit three major types of substrate specificities, which are determined by recognition of the P1 substrate residue (the amino acid residue preceding the cleavage site) by the $\mathrm{S} 1$ pocket of the enzyme. The trypsin-type prefers positively charged residues at the P1 position, whereas the chymotrypsin-type and the elastase-type prefer large and small hydrophobic residues, respectively ${ }^{36,37}$. To gain more information 
Fig. 2 FAM111A binds to ssDNA through its central region. a Electrophoretic mobility shift assay (EMSA). DNA substrates labeled with IRDye700 (ss: single-stranded, ds: double-stranded, $Y$ Y fork, ds $Y$ double-stranded $Y$ fork) were incubated with increasing amounts of recombinant GST-FAM111A-strep protein $(50,150$, and $450 \mathrm{nM})$. BSA ( $450 \mathrm{nM})$ was added in the "-" lanes. Positions of the shifted band are indicated by arrowheads. *, nonspecific band. b Supershift assay. GST-FAM111A-strep $(450 \mathrm{nM})$ was pre-incubated with anti-FAM111A antibody on ice for 30 min before adding ssDNA oligos. Positions of the shifted and supershifted bands are indicated by a black and a white arrowhead, respectively. ${ }^{\star}$, nonspecific band. $\mathbf{c}$ Fluorescence polarization (FP) DNA-binding assay. Various amounts of recombinant strep-FAM111A protein or BSA were mixed with 6-FAM-labeled ssDNA oligos and FP values were measured. Values are mean \pm s.d. of independent experiments $(n=3)$. d Schematic representation of full-length (FL) and truncated FAM111A proteins used in this assay. Ability to bind to ssDNA oligos is summarized on right. PIP: PCNA-interacting peptide box. e Mapping the DNA-binding domain by EMSA using FAM111A fragments. IRDye700-labeled ssDNA oligos were incubated with increasing amounts of recombinant MBP-FAM111A proteins indicated on top $(50,150,450$, and $1350 \mathrm{nM})$. MBP (1350 nM) was added in the "-" lane. $\mathbf{f}$ Effect of the F231A mutation on ssDNA binding. Increasing amounts of MBPFAM111A 1-282 proteins indicated on top (150, 450, $1350 \mathrm{nM}$ for WT and equivalent amounts for F231A) were incubated with IRDye700-labeled ssDNA (left) or Y-fork (right) oligos. MBP (1350 nM) was added in the "-" lane. a, b, e, f Percentages of band shifts in each lane are shown below. Red dots indicate IRDye700. Source data are provided as a Source Data file.

on the substrate specificity of the in vivo FAM111A cleavage, we set out to determine the in vivo cleavage site of FAM111A. We expressed recombinant FAM111A proteins in insect cells and purified with the C-terminal Strep-tag. As was the case with FAM111A expressed in human cells, recombinant FAM111A produced in insect cells displayed a processed product that was more prominent with the FAM111A R569H mutant and absent when the active site was mutated (Fig. 3c). N-terminal peptide sequencing of the C-terminal fragment from the FAM111A $\mathrm{R} 569 \mathrm{H}$ mutant identified the cleavage site to be C-terminal to Phe334 (P1) (Fig. 3d). Cleavage at this site is predicted to produce $38.4 \mathrm{kDa} \mathrm{N}$-terminal and $31.5 \mathrm{kDa} \mathrm{C}$-terminal fragments, consistent with the observed fragment sizes (Fig. 3a-c). Substitution of Phe334 with glycine or arginine diminished the in vivo cleavage of FAM111A (Fig. 3e), suggesting that Phe334 contributes to the specificity of the cleavage site. The hydrophobic residue (Phe334) at the P1 position implies chymotrypsin-type specificity for this cleavage.

Chymotrypsin-like proteases typically contain a hydrophobic S1 pocket ${ }^{36,37}$. In examining the structure model of the FAM111A trypsin-like domain based on the plant Deg1 protease $^{38,42}$, we indeed found a notable hydrophobic feature, Phe536, which is highly conserved among species, in the predicted S1 pocket (Supplementary Fig. 4b). In silico docking experiments using peptide sequences with the native cleavage site RTTFGKV as well as the mutant cleavage sites RTTRGKV and RTTGGGKV (underlines indicate the P1 residue) showed the P1 Phe substrate displayed by far better docking score $(-12.9 \mathrm{kcal}$ $\mathrm{mol}^{-1}$ ) compared with either the P1 Arg or P1 Gly substrates $\left(-10.5\right.$ and $-8.5 \mathrm{kcal} \mathrm{mol}^{-1}$, respectively). Close examination of the $\mathrm{S} 1$ pocket revealed that the docked Phe peptide resulted in an edge-to-face ring stacking interaction between the P1 Phe334 and conserved Phe536 (Fig. 3f). By contrast, in the docking experiment with the P1 Arg mutant peptide, the Arg side chain was expelled from the $\mathrm{S} 1$ pocket during the energy minimization and relaxation protocol. These observations indicate that FAM111A is a serine protease with chymotrypsin-like specificity and strongly suggest that FAM111A exhibits autocleavage activity in vivo.

FAM111A autocleavage can occur in trans. Autocleavage can occur intramolecularly (in cis) and intermolecularly (in trans). Co-immunoprecipitation assays using FAM111A with different tags (3xFlag or EGFP) showed interaction between 3xFlagFAM111A and EGFP-FAM111A (Fig. 3g), raising the possibility of an intermolecular mechanism. To directly assess this possibility, we co-expressed the constitutively active form of FAM111A (R569H) and its inactive counterpart (R569H/S541A) with different tags and tested whether constitutively active FAM111A causes cleavage of inactive FAM111A. Indeed, cleavage of the inactive FAM111A occurred when the constitutively active FAM111A was co-expressed (3xFlag-FAM111A R569H/S541A with EGFP-FAM111A R569H and vice versa) (Fig. 3h, lanes 6 and 7), demonstrating that autocleavage of FAM111A can occur intermolecularly.

FAM111A autocleavage requires an intact DNA-binding domain. We next determined whether domains other than the trypsin-like domain are required for the FAM111A autocleavage. Introduction of the F231A mutation, which disrupts ssDNA binding of FAM111A (Fig. 2f, Supplementary Fig. 3j-l), but not YFAA, which diminishes PCNA interaction ${ }^{34}$, prevented autocleavage of the constitutively active form of FAM111A (R569H) (Fig. 3i). Co-immunoprecipitation assays ruled out the possibility that the F231A and S541A mutations disrupted intermolecular interaction (Supplementary Fig. 4c). These data suggest that FAM111A activity in vivo is dependent on an intact ssDNAbinding domain.

Trapping-dependent PARPi hypersensitivity after FAM111A KO. In principle, PARPis can affect cell proliferation and survival by (i) diminishing formation of poly(ADP-ribose) polymer (inhibition mechanism) and (ii) blocking DNA replication through PARP1-DNA complex formation (trapping mechanism). The inhibition mechanism would be mimicked by PARP1 KO, while the trapping mechanism would be eliminated by PARP1 KO. To test which mechanism underlies the PARPi hypersensitivity conferred by FAM111A KO, we knocked out PARP1 by CRISPR/Cas9 in the FAM111A WT and FAM111A KO backgrounds (Fig. 4a, Supplementary Fig. 1 and Supplementary Table 1). FAM111A/PARP1 double KO (DKO) cells, as well as $P A R P 1 \mathrm{KO}$ cells, were viable and indistinguishable from parental cells in proliferation assays (Fig. 4b). These data argue against PARP1 inhibition being the major mechanism of the hypersensitivity to PARPis in FAM111A KO cells. On the other hand, $P A R P 1$ knockout conferred complete niraparib resistance to both FAM111A WT and FAM111A KO cells (Fig. 4c). Taken all together, these data suggest that sensitization to PARPis by FAM111A $\mathrm{KO}$ is dependent on PARP1 trapping.

FAM111A deficiency causes TOP1cc accumulation. The hypersensitivity of FAM111A KO cells to CPT (Fig. 1a, b) prompted us to hypothesize that FAM111A might also be involved in DPC repair in vivo. We tested this possibility by examining the levels of TOP1cc in FAM111A KO cells in the absence of CPT treatment. We found that FAM111A KO cells displayed increased TOP1cc foci compared with wild-type cells (Fig. 4d-f). Increased TOP1cc foci were also observed in U2OS cells after knockdown of FAM111A by RNAi (Supplementary Fig. 5a-c). TOP1cc accumulation in the FAM111A KO cells was 
a

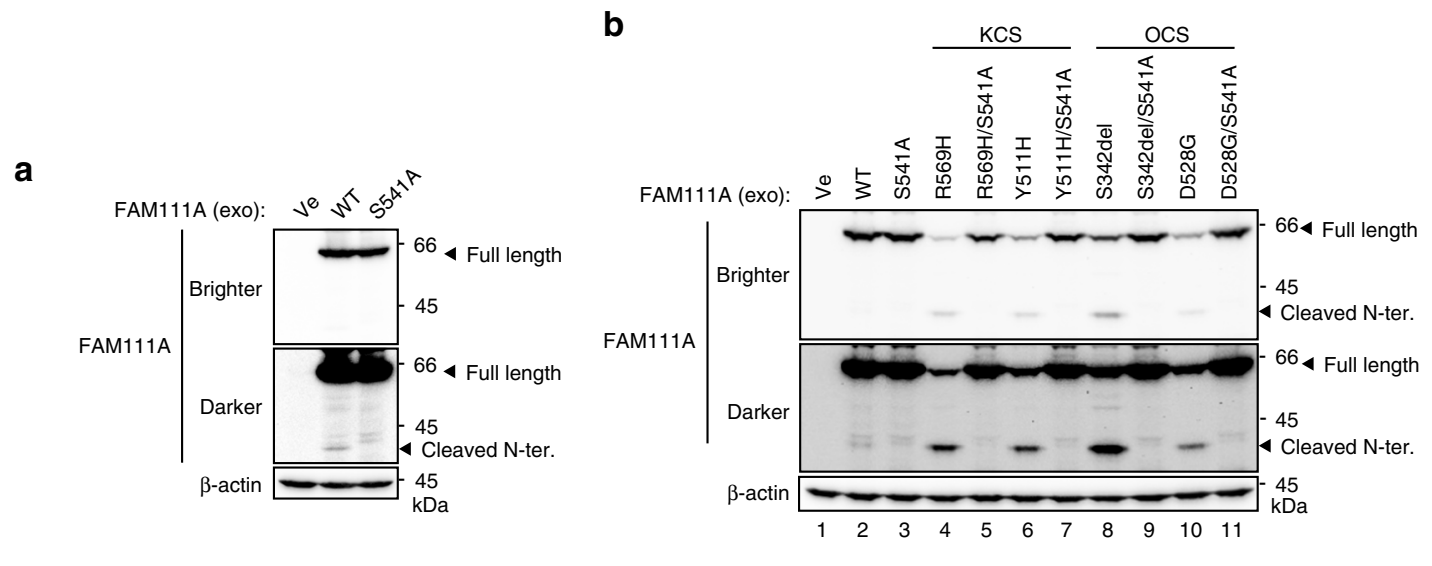

b
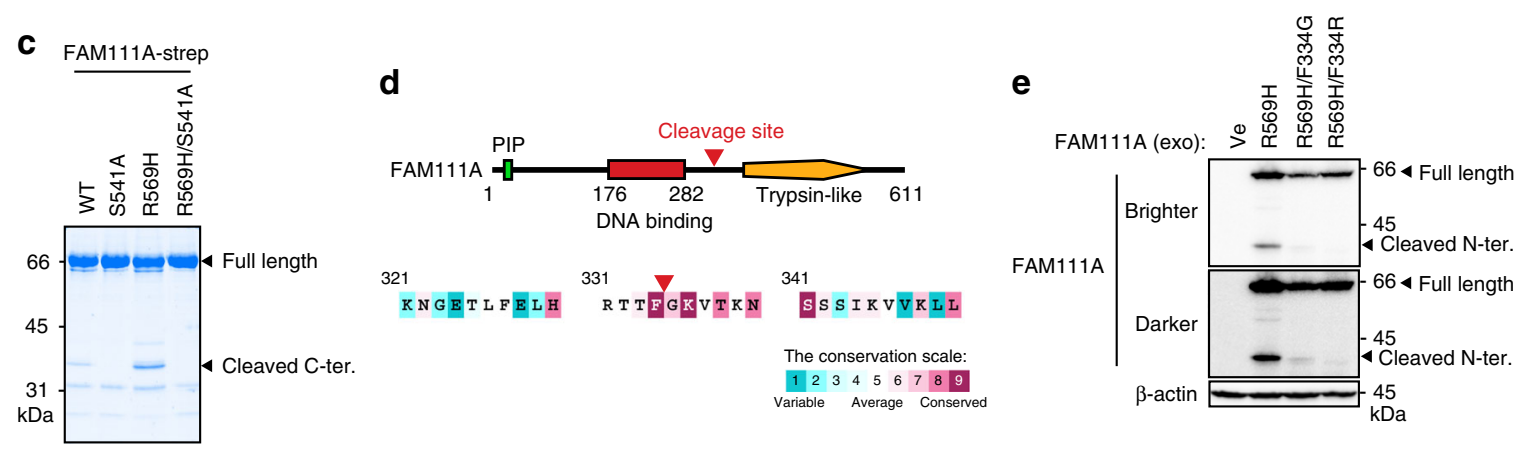

f

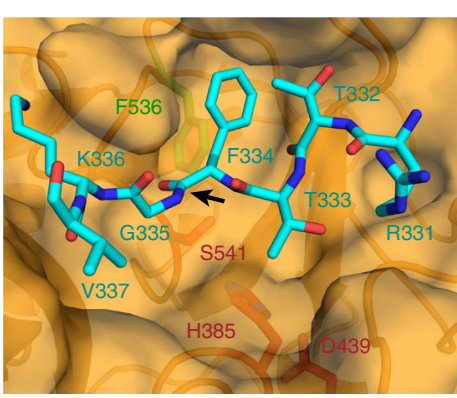

g
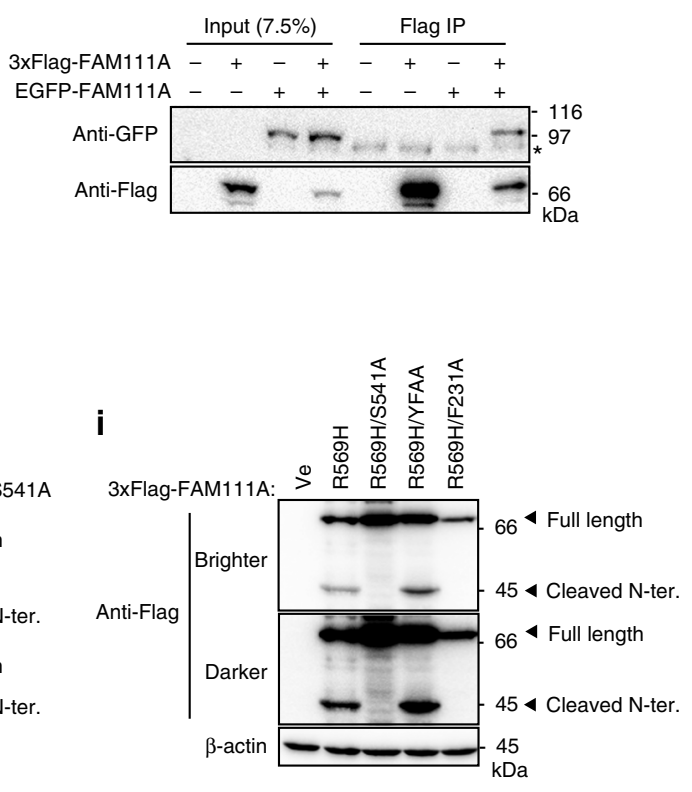

diminished by exogenous expression of wild-type FAM111A, but not the active site (S541A) or the PIP-box (YFAA) mutants (Fig. 4d-f), indicating the importance of these domains to prevent TOP1cc accumulation. These results indicate that FAM111A is important for the repair of TOP1ccs, providing an explanation for the hypersensitivity of FAM111A KO cells to CPT.

FAM111A prevents fork stalling during PARPi or CPT treatment. Given that FAM111A localizes to replication forks ${ }^{34}$, we assessed the importance of FAM111A in protecting replication forks from protein obstacles imposed by CPT or PARPis. For these experiments, we employed DNA combing assays and examined the effect of the drugs on replication fork movement. Nascent DNA was sequentially labeled with CldU and then with IdU in the presence or absence of drugs (Fig. 5a). In the absence of drug treatment, replication fork movement was largely unaffected by FAM111A KO as seen in the comparable CldU tract lengths in FAM111A WT and KO cells (Fig. 5b). Although it has been reported that FAM111A may be involved in the PCNA loading ${ }^{34}$, the amounts of PCNA on chromatin were largely unaffected in FAM111A KO cells (Supplementary Fig. 6a). In contrast, replication fork movement was severely affected by niraparib or CPT treatment in FAM111A KO cells as evidenced 
Fig. 3 In vivo cleavage of FAM111A. a Detection of a cleaved FAM111A fragment. FAM111A proteins (WT or S541A) were transiently expressed in 293T cells and the indicated proteins were analyzed by Western blotting. Positions of the full-length and cleaved $\mathrm{N}$-terminal fragment ( $\mathrm{N}$-ter.) are indicated. $\mathbf{b}$ Effect of mutations found in Kenny-Caffey syndrome (KCS) and osteocraniostenosis (OCS) on FAM111A cleavage. FAM111A proteins (WT or indicated mutants) were transiently expressed in 293T. The indicated proteins were analyzed by Western blotting. c Recombinant FAM111A-strep proteins. WT or indicated mutants of FAM111A-strep proteins were produced in insect cells and purified by the C-terminal strep tag. Positions of the full-length and cleaved C-terminal fragment (C-ter.) are indicated. $\mathbf{d}$ Identification of the FAM111A cleavage site by N-terminal Edman degradation. The position of the cleavage site is indicated in the schematic representation of FAM111A (upper) and in the amino acid sequence of FAM111A (lower). The color scale of the degree of amino acid conservation among species is shown below. e Effect of amino acid substitutions at the P1 site (Phe334) on in vivo cleavage of FAM111A R569H. The indicated FAM111A proteins were transiently expressed in 293T cells. Indicated proteins were detected by Western blotting. f Docked model of cleavage site residues (cyan) bound in the active site of FAM111A trypsin-like domain (orange). Catalytic triad residues are labeled in red and cleavage site is indicated by a black arrow. $\mathbf{g}$ Co-immunoprecipitation assays. 3xFlag-FAM111A and EGFP-FAM111A were transiently expressed in $293 \mathrm{~T}$ as indicated and anti-Flag immunoprecipitation was performed. Input and precipitated proteins were analyzed by Western blotting. ${ }^{*}$, nonspecific band. $\mathbf{h}$ Trans-cleavage assays. 3xFlag-tagged and EGFP-tagged mutant FAM111A proteins (R: R569H, R/S: R569H/S541A) were transiently expressed in 293 T cells. Expressed proteins were analyzed by Western blotting. i Effect of the mutations in the putative active site (S541A), PIP box (YFAA) and DNAbinding domain (F231A) on in vivo cleavage of FAM111A R569H. The indicated 3xFlag-FAM111A proteins were transiently expressed in 293T cells. Expressed proteins were analyzed by Western blotting. Empty vectors were used in "Ve" or "-" lanes.

by the reduced ratios of IdU- and CldU-labeled tract lengths (Fig. 5c, d). Exogenous expression of wild-type FAM111A, but not the S541A, or YFAA, rescued the effect of niraparib treatment in FAM111A KO cells (Fig. 5e), suggesting that the putative protease activity and PIP box of FAM111A are important to mitigate the effect of trapped PARPs. In addition, the F231A mutant did not rescue the replication fork defects during niraparib or CPT treatments (Fig. 5f, g), demonstrating the importance of the FAM111A DNA-binding domain. Importantly, the percentage of collapsed forks induced by hydroxyurea (HU) treatment was not increased in FAM111A KO cells (Supplementary Fig. 6b, c), suggesting that FAM111A does not regulate the stability of stalled replication forks. In addition, the S541A and F231A mutants of FAM111A localized properly to replication forks as assessed by iPOND assays (Supplementary Fig. 6d). These results demonstrate the importance of FAM111A in overcoming protein obstacles imposed by CPT and PARPis.

We reported previously that Sprtn hypomorphic MEFs are hypersensitive to CPT but not niraparib ${ }^{27}$. Together with the drug sensitivity data presented above (Fig. 1a, b), the previous observations raised the possibility that SPRTN might engage with TOP1cc but not PARP1-DNA complexes, while FAM111A might act on both. To investigate this possibility, we targeted SPRTN with CRISPR/Cas9 in HAP1, the same human cell line as FAM111A KO cells. This resulted in a SPRTN hypomorphic cell line (indicated as SPRTN CRISPR), which exhibited greatly reduced SPRTN levels due to $\mathrm{KO}$ in one allele and an eightamino-acid deletion in the metalloprotease domain in the other allele (Fig. 5h, Supplementary Fig. 1 and Supplementary Table 1). Consistent with our previous study in mouse cells ${ }^{27}$, the SPRTN CRISPR cells were sensitive to CPT, validating impairment of SPRTN function, yet they were not hypersensitive to niraparib (Supplementary Fig. 6e). DNA combing assays revealed that SPRTN CRISPR cells exhibited increased replication fork stalling with CPT but not with niraparib, whereas FAM111A KO displayed fork stalling with both drugs (Fig. 5i). These results demonstrate the unique function of FAM111A in processing PARP1-DNA complexes.

FAM111A KO causes enhanced DNA damage after PARPi treatment. Finally, we investigated how FAM111A KO confers increased PARPi sensitivity and cell death (Fig. 1). If increased replication fork stalling (Fig. 5c) is the underlying cause, FAM111A KO cells are expected to experience cell-cycle defects and increased levels of DNA damage after PARPi treatment. Consistent with this prediction, FAM111A KO cells, which showed a modest reduction of G1 phase and increase in $S$ and
G2/M phases in the absence of drug treatment, exhibited profound accumulation in G2/M phase upon treatment with niraparib (Fig. 6a, b). Moreover, FAM111A KO cells exhibited higher levels of phospho-Chk1, but not phospho-Chk2, than wild-type cells after niraparib treatment (Fig. 6c), indicating augmented activation of the ATR/Chk1 pathway, which typically responds to DNA replication stress. In addition, higher levels of DNA damage were observed in FAM111A KO cells than in wild-type cells after treatment with niraparib, as shown by immunostaining with the DNA damage marker $\gamma \mathrm{H} 2 \mathrm{AX}$ (Fig. 6d, e). Collectively, these results suggest that the absence of FAM111A causes replication fork stalling in S phase after PARPi treatment, leading to DNA damage, cell-cycle arrest in G2/M phases, and eventually cell death.

\section{Discussion}

Advancing DNA replication forks face many obstacles due to proteins that are covalently crosslinked or tightly bound to DNA. In this study, we discovered that the replication fork protein FAM111A is important for resolving replication stalling caused by protein obstacles. We provide evidence for the protease activity of FAM111A in vivo, which is dependent on the DNAbinding domain identified in this study (Figs. 2 and 3). FAM111A KO cells exhibit TOP1cc accumulation and PARPi hypersensitivity that is dependent on PARP-trapping (Fig. 4). In the absence of FAM111A, protein obstacles induced by a TOP1 poison or a PARPi causes increased replication stalling (Fig. 5). The TOP1cc removal and fork protection by FAM111A require the PIP box and an intact trypsin-like domain (Figs. 4 and 5). Collectively, we propose a model that the FAM111A PIP box tethers the trypsinlike protease domain to replication forks to protect them from protein obstacles (Fig. 6f).

The role of FAM111A in resolving protein obstacles at replication forks is reminiscent of SPRTN's function in replication-coupled DPC repair. Both proteins contain a protease domain, a PIP box, and a DNA-binding domain that is necessary for protease activation. In addition, both proteins localize to replication forks and facilitate DNA replication at DPCs, including TOP1ccs induced by CPT. Despite these similarities, however, a striking difference was noted between FAM111A and SPRTN in the response to PARPis. Whereas CPT induces protein obstacles covalently crosslinked to DNA, PARPis are generally thought to trap PARPs non-covalently ${ }^{9-13}$, although covalent PARP1-DNA complexes have also been reported ${ }^{43,44}$. Interestingly, it was recently demonstrated in Xenopus egg extracts that SPRTN activation requires polymerase collision with DPCs followed by helicase bypass of DPCs ${ }^{29,45}$. 
a

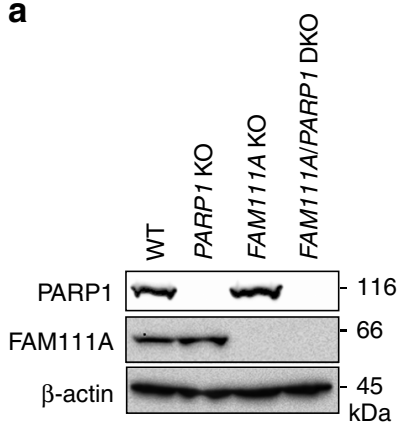

b

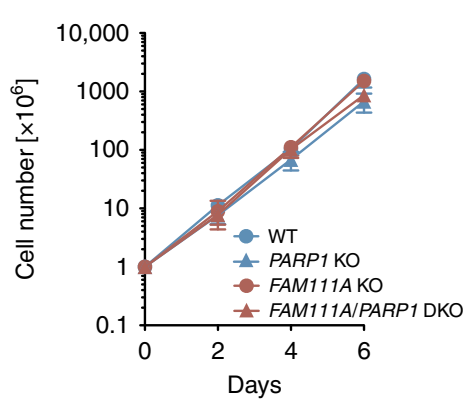

c

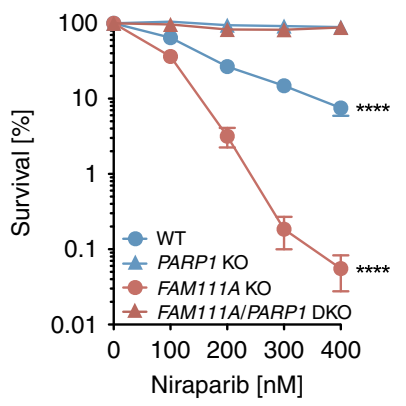

d

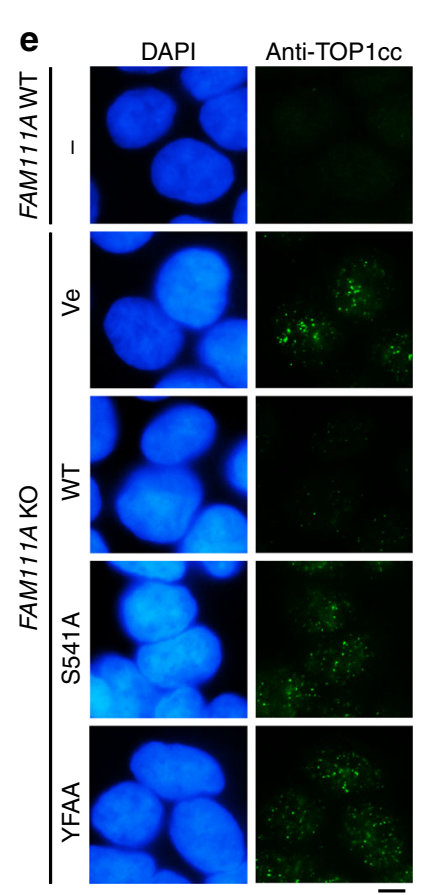

Fig. 4 FAM111A KO causes trapping-dependent PARPi sensitization and TOP1cc accumulation. a Knockout of PARP1 by CRISPR/Cas9. Parental HAP1 (WT), PARP1 KO \#17, FAM111A KO \#14, FAM111A/PARP1 double knockout (DKO) \#1 were analyzed for expression of the indicated proteins by Western blotting. $\mathbf{b}$ Cell proliferation assays. Cells analyzed in a were cultured and cell numbers were counted every other day up to 6 days. Values are mean \pm s.d. of independent experiments $(n=3)$. c Clonogenic survival assays. Cells analyzed in a were cultured in the presence of the indicated concentrations of niraparib for 6 days. Results shown are representative of three independent experiments and values represent mean $\pm \mathrm{s}$.d. of technical replicates $(n=3)$. $p$ values were calculated between WT and PARP1 KO and between FAM111A KO and FAM111A/PARP1 DKO, respectively. ${ }^{\star \star \star \star} p<0.0001$ (two-tailed unpaired $t$-test). $\mathbf{d}$ Exogenous (exo) expression of WT and mutant FAM111A proteins in FAM111A KO cells. The indicated FAM111A proteins were stably expressed by lentiviral vectors in FAM111A KO \#14 and analyzed by Western blotting. Parental HAP1 (WT) is shown as a reference. Ve empty vector. e TOP1cc focus formation. Cells analyzed in d were stained with anti-TOP1cc antibody and DAPI. Scale bar, $5 \mu \mathrm{m}$. $\mathbf{f}$ Quantification of cells containing TOP1cc foci. Experiments were performed as in e. At least 100 cells were scored by an investigator blinded to sample identity and percentages of cells with 8 or more foci are shown. Values are mean \pm s.d. of independent experiments $(n=3) .{ }^{\star \star *} p<0.001$; n.s. not significant (two-tailed unpaired $t$-test). Source data are provided as a Source Data file.

Therefore, the difference between SPRTN and FAM111A in the response to PARPis might be attributable to whether the forkblocking proteins are covalently crosslinked to DNA or not. SPRTN might be specialized for protein obstacles covalently bound to DNA, while FAM111A might have a broader role in responding to protein obstacles regardless of the DNAcrosslinking status. Although further investigations are necessary to understand how different types of protein obstacles are cleared at replication forks, our study suggests that cells are equipped with multiple mechanisms to deal with various protein obstacles during DNA replication.

Our study strongly suggests that FAM111A functions as a protease in vivo. A mutation in the putative active site serine abolishes its function in TOP1cc repair and replication during niraparib treatment (Figs. $4 \mathrm{~d}-\mathrm{f}$ and 5e). Although wild-type FAM111A shows limited protease activity when expressed in cells, the FAM111A mutants found in KCS or OCS patients are constitutively active and display increased autocleavage activity in vivo (Fig. 3b). We speculate that FAM111A might undergo activation only when necessary, and the KCS/OCS mutants might have bypassed the required activation mechanism. Homologybased structure modeling suggests that the FAM111A trypsin-like domain is similar to the Deg/HtrA family proteases ${ }^{38}$, which contain one or two PDZ domains that play regulatory roles during protease activation in response to various cellular stresses, including high temperature, abnormal $\mathrm{pH}$, or unfolded proteins ${ }^{42,46,47}$. Although FAM111A lacks a PDZ domain, we instead identified a DNA-binding domain in FAM111A that is necessary for FAM111A activity in vivo (Figs. 2 and 3i). This raises the possibility that FAM111A might undergo activation by sensing ssDNA structures as has been demonstrated for SPRTN. However, ssDNA-binding alone might not be a sufficient 
a

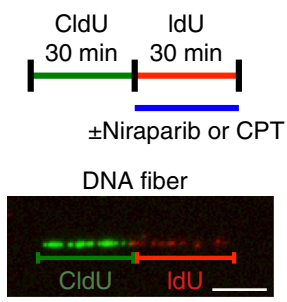

b

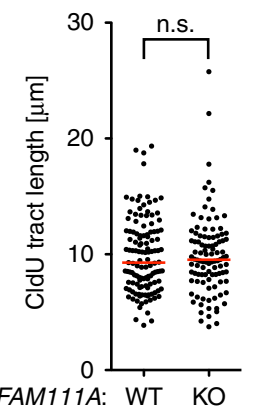

$r^{\prime \prime 2} n^{n}, n^{2}$

C

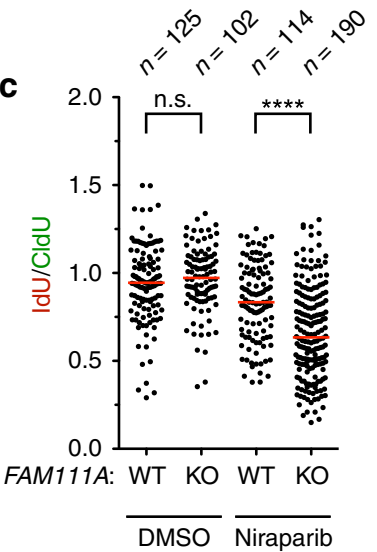

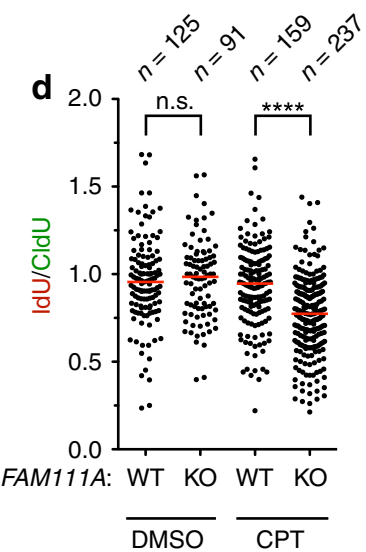

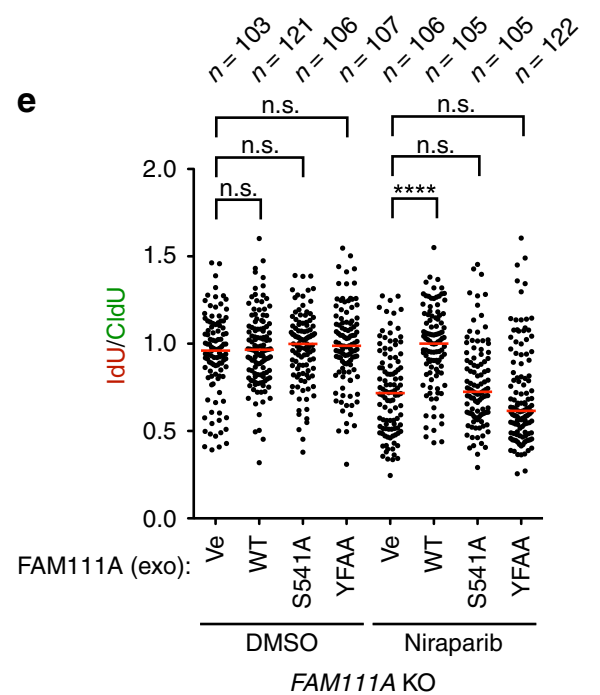

FAM111A KO $\mathbf{h}$

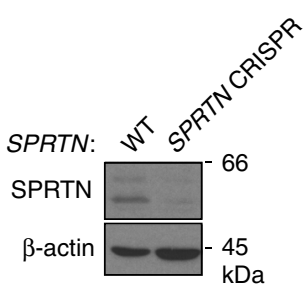

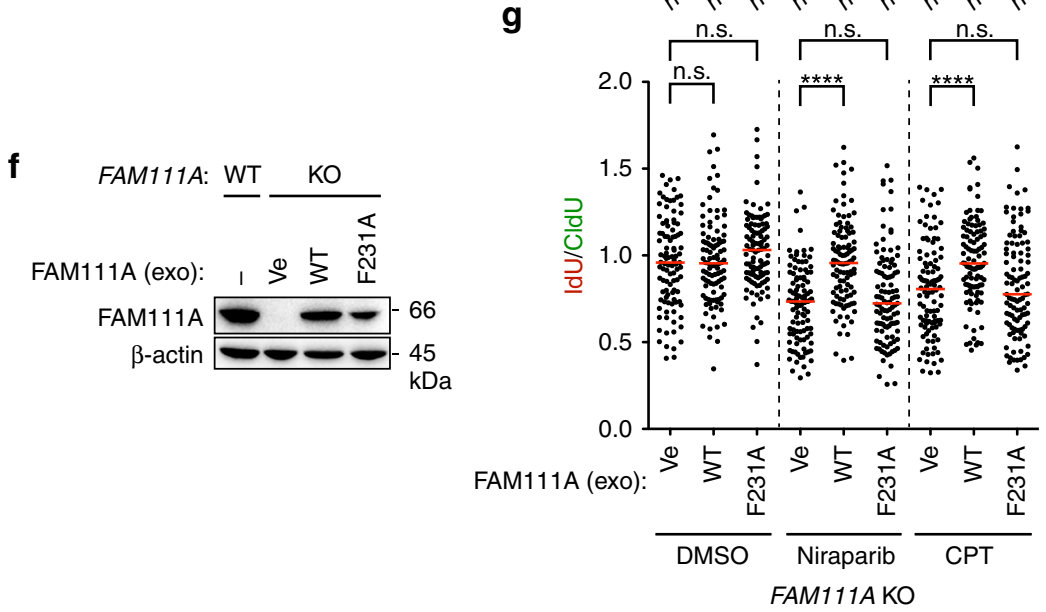

g

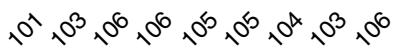

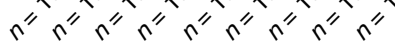

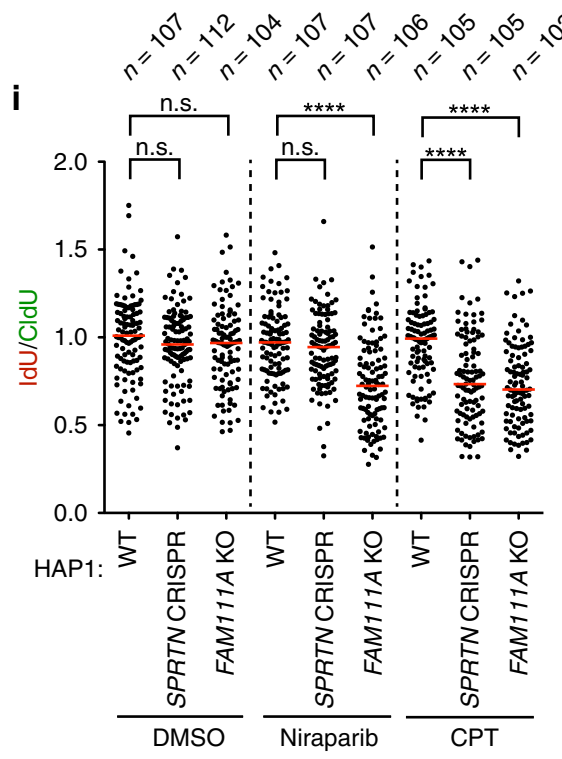

mechanism to restrict FAM111A activation to events of replication fork collisions with protein obstacles. Consistent with this notion, we have not been able to demonstrate protease activities in vitro using purified recombinant FAM111A even in the presence of multiple DNA structures. One possibility is that FAM111A might undergo additional regulation in vivo to become fully active, for example, through post-translational modifications, cofactor binding, or conformational changes. Interestingly,

many Deg/HtrA proteases dramatically change their oligomer status during activation, in some cases forming cages with the protease active sites facing inward ${ }^{47,48}$. Indeed, our results showed that FAM111A proteins interact with each other in vivo (Fig. 3g). While the activation mechanism is still unclear, our study suggests that FAM111A might be a stress-responsive protease similar to the Deg/HtrA family proteases, but one that responds to replication stress imposed by protein obstacles. 
Fig. 5 FAM111A is important to prevent replication fork stalling during PARP and TOP1 inhibitor treatment. a Schematic representation of DNA combing assays. Nascent DNA was labeled with CldU (30 min) followed by IdU (30 min). Where indicated, niraparib or CPT was added during IdU labeling (upper panel). A representative picture of a replication track is shown (lower panel). Scale bar, $5 \mu \mathrm{m}$. b A dot plot showing the length of nascent DNA labeled by CldU in parental HAP1 (WT) and FAM111A KO \#14. Distribution of the tract length is shown. $\mathbf{c}, \mathbf{d}$ DNA combing assays. Parental HAP1 (WT) and FAM111A KO \#14 were treated with $3 \mu \mathrm{M}$ niraparib (c) or $30 \mathrm{nM} \mathrm{CPT} \mathrm{(d)} \mathrm{during} \mathrm{IdU} \mathrm{labeling.} \mathrm{Distribution} \mathrm{of} \mathrm{replication} \mathrm{forks} \mathrm{at} \mathrm{different} \mathrm{IdU/CldU} \mathrm{ratios} \mathrm{is}$ shown. e DNA combing assays. FAM111A KO \#14 expressing various FAM111A proteins (Fig. 4d) were treated with or without $3 \mu M$ niraparib during IdU labeling. Distribution of replication forks at different IdU/CIdU ratios is shown. $\mathbf{f}$ Exogenous (exo) expression of WT and mutant FAM111A proteins in FAM111A KO cells. The indicated FAM111A proteins were stably expressed by lentiviral vectors in FAM111A KO \#14 and analyzed by Western blotting. Parental HAP1 (WT) is shown as a reference. $\mathbf{g}$ DNA combing assays. FAM111A KO \#14 cells expressing WT or the DNA-binding domain mutant (F231A) were treated with $3 \mu \mathrm{M}$ niraparib or $30 \mathrm{nM}$ CPT during IdU labeling. Distribution of replication forks at different IdU/CldU ratios is shown. $\mathbf{h}$ Depletion of SPRTN by CRISPR/Cas9 in HAP1 cells. The indicated proteins were analyzed by Western blotting. i DNA combing assays. Parental HAP1 (WT), SPRTN CRISPR \#8 and FAM111A KO \#14 were treated with $3 \mu \mathrm{M}$ niraparib or $30 \mathrm{nM} \mathrm{CPT} \mathrm{during} \mathrm{IdU} \mathrm{labeling.} \mathrm{Distribution} \mathrm{of} \mathrm{replication} \mathrm{forks} \mathrm{at} \mathrm{different} \mathrm{IdU/CldU}$ ratios is shown. $\mathbf{b}-\mathbf{e}, \mathbf{g}$, $\mathbf{i}$ Replication tracts were measured by an investigator blinded to sample identity. Horizontal red lines indicate median values and $p$ values were obtained by two-tailed unpaired $t$-test $\left({ }^{\star \star \star \star} p<0.0001 ; n . s\right.$. not significant). $n$ is the number of individual measures. Ve empty vector. Source data are provided as a Source Data file.

The present study also identifies FAM111A deficiency as a mechanism of hypersensitivity to PARPis. Because FAM111A deficiency augments DNA damage induced by PARPis (Fig. 6d, e), FAM111A inhibition might be a viable strategy to enhance PARPi killing in HR-deficient tumors and to expand PARPi therapies to HR-proficient tumors. Moreover, our data showed that FAM111A levels vary among cancer cell lines (Supplementary Fig. 4a). Therefore, determining the FAM111A status in tumors might be useful because a therapeutic advantage can be gained through the selective vulnerability of FAM111A-deficient cells to PARPis.

Finally, the present results shed important new light on previous studies of FAM111A. Our data suggest that FAM111A mutations found in KCS or OCS patients lead to constitutive protease activity (Fig. 3b). This observation raises two possibilities for the potential consequences of disease-associated mutations. One is that hyperactivation of FAM111A causes abnormal degradation of DNA-binding proteins in KCS and OCS, and the other is that hyperactivation causes a FAM111A deficiency through hyper-autocleavage. In fact, FAM111A forms oligomers and can degrade FAM111A proteins in trans (Fig. $3 \mathrm{~g}, \mathrm{~h}$ ), so that it is possible that the heterozygous mutations in KCS and OCS cause depletion of total FAM111A protein through a dominant-negative effect. Another implication of our study is that it might help understand the role of FAM111A as an antiviral defense mechanism. It has been demonstrated that FAM111A is a host range restriction factor for SV40 and mutant orthopoxviruses ${ }^{49,50}$. Given the recent report showing that FAM111A localizes to SV40 viral replication centers ${ }^{51}$, it is possible that FAM111A might restrict viral replication by recognizing and degrading viral nucleoprotein complexes.

In conclusion, we demonstrated that FAM111A is important for DNA replication at protein obstacles. This study not only sets new frameworks for future studies on DPC repair, replication fork regulation, and anti-cancer therapies, but also provides new insight into the role of FAM111A in genetic diseases and viral defense mechanisms.

\footnotetext{
Methods

Cell culture. Human chronic myelogenous leukemia cell line HAP1 was purchased from Horizon Discovery. Human embryonic kidney cell line 293T, human osteosarcoma cell line U2OS, human proximal tubular cell line HK2, human lung fibroblast cell line MRC-5, human cervical adenocarcinoma cell line HeLa, human colorectal carcinoma cell line HCT116, human mesothelioma cell line H226, human fibrosarcoma cell line HT1080, and human hepatocellular carcinoma cell line HepG2 were obtained from American Type Culture Collection. These cell lines were cultured in respective medium: Iscove's Modified Dulbecco's Medium for HAP1, Dulbecco's modified Eagle's medium for 293T and HeLa, McCoy's 5A medium for U2OS and HCT116, Eagle's minimum essential medium for MRC-5, HT1080, and HepG2. All of these media were supplemented with $10 \%$ fetal bovine serum (FBS). For HT1080 and HepG2, the medium was also supplemented with
}

MEM non-essential amino acids (Corning) and sodium pyruvate (Corning). HK2 cell line was cultured in keratinocyte serum free medium (Invitrogen).

Expression Plasmids. For mammalian cell expression, a cDNA fragment encoding human FAM111A was cloned in the following vectors: pLVX2-IRES-puro (no epitope tag), pLVX3-IRES-puro (for an N-terminal 3xFlag tag), and pLVX6-IRES puro (for an N-terminal EGFP tag). For insect cell expression, a DNA fragment encoding FAM111A with tandem Strep tags (FAM111A-strep) was synthesized with codon-optimization for expression in S. frugiperda (Thermo Fisher Scientific) (Supplementary Table 2). A DNA fragment encoding FAM111A with an Nterminal tandem Strep-tag (Strep-FAM111A) was generated by PCR. The ORFs were cloned into pDEST20 (for an N-terminal GST tag) and pFastBac NT-B. For bacterial expression, a DNA fragment encoding FAM111A 1-282 was generated by gene synthesis with codon-optimization for expression in E. coli (Thermo Fisher Scientific) (Supplementary Table 2) and used as a PCR template to amplify DNA fragments for FAM111A 1-99, 100-175, and 176-282. A DNA fragment encoding FAM111A 283-611 was amplified from human FAM111A cDNA. These PCR fragments were cloned in pMALII-c2x for expression of MBP fusion proteins in $E$ coli. Point mutations were introduced using the Quickchange mutagenesis kit (Agilent) or by PCR. All plasmids were confirmed by Sanger sequencing. Cloning primers are summarized in Supplementary Table 3.

Transfection and viral infection. Plasmid transfection was performed using Lipofectamine 2000 (Thermo Fisher Scientific) or Turbofectin 8.0 (OriGene) according to suppliers' instructions. Lentiviruses were produced by transfecting 293 T cells with a lentiviral and two packaging plasmids (psPax2 and pMD2.G). Culture media containing lentiviral particles were harvested after $48 \mathrm{~h}$ and used to infect cells in the presence of $4 \mu \mathrm{g} \mathrm{ml}^{-1}$ polybrane. After selection with $2 \mu \mathrm{g} \mathrm{ml}^{-1}$ puromycin for $48 \mathrm{~h}$, cells were cultured in the absence of puromycin for at least $24 \mathrm{~h}$. siRNA transfection was performed using RNAiMAX (Thermo Fisher Scientific). The target sequences of siRNAs are as follows: siControl, 5'-CGUACGCG GAAUACUUCGA-3'; siFAM111A.271, 5'-CUAAAGAGCAACAGAAUAA-3'; siFAM111A.1213, 5'-CGAUUAAAGUAGUGAAACU-3'. siRNA oligo sequences are shown in Supplementary Table 3.

Western blotting and co-immunoprecipitation. Cells were lysed using NP-40 lysis buffer (50 mM Tris- $\mathrm{HCl} \mathrm{pH} 7.4,150 \mathrm{mM} \mathrm{NaCl}, 0.1 \% \mathrm{NP}-40,10 \%$ glycerol, $5 \mathrm{mM}$ EDTA, $50 \mathrm{mM} \mathrm{NaF}, 1 \mathrm{mM} \mathrm{Na} \mathrm{VO}_{4}$ ) supplemented with protease inhibitor cocktail (Sigma). For FAM111A autocleavage assays, cells were lysed in SDS lysis buffer (PBS supplemented with 1\% SDS and protease inhibitor cocktail). Cell lysates containing $30 \mu \mathrm{g}$ protein were run on an SDS-PAGE gel and analyzed by Western blotting. Primary antibodies used were rabbit anti-FAM111A (Abcam, ab184572, 1:2000), rabbit anti-FAM111A (Sigma, HPA040176, 1:500), mouse antiPARP1 (C-2-10, kind gift from Guy Poirier, 1:1000), mouse anti-SPRTN ${ }^{52}$, mouse anti- $\beta$-actin (Sigma, A5316, 1:5000), rabbit anti-Chk1 (Epitomics, 1740-1, 1:2000), rabbit anti-phospho-Chk1 (S345) (Cell Signaling Technology, \#2348, 1:1000), mouse anti-Chk2 (BD Bioscience, 611570, 1:1000), rabbit anti-phospho-Chk2 (T68) (Cell Signaling Technology, \#2197, 1:1000), mouse anti-PCNA (Santa Cruz, sc-56, 1:2000), rabbit anti-Histone H3 (Cell Signaling Technology, \#9715, 1:1000), mouse anti-Flag (Sigma, F1804, 1:1500), rabbit anti-Flag antibody (Cell Signaling Technology, \#2368, 1:1000), and mouse anti-GFP (Santa Crus, sc-9996, 1:1000). Chemiluminescence signals were imaged by X-ray film exposure or ChemiDoc (BioRad)

For co-immunoprecipitation experiments, 293T cells were transfected with expression plasmids for $48 \mathrm{~h}$ and lysed with NP-40 lysis buffer supplemented with proteinase inhibitor cocktail (Sigma) and $41 \mathrm{U} \mathrm{ml}^{-1}$ Benzonase (Novagen). Cell lysates containing $\sim 1 \mathrm{mg}$ protein were incubated with anti-Flag beads (Sigma) for 
a

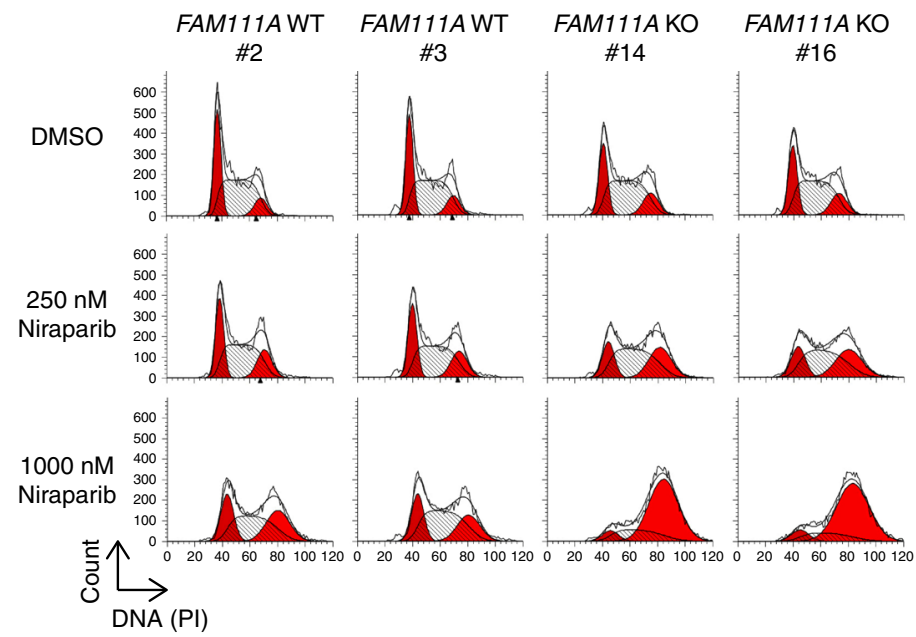

b

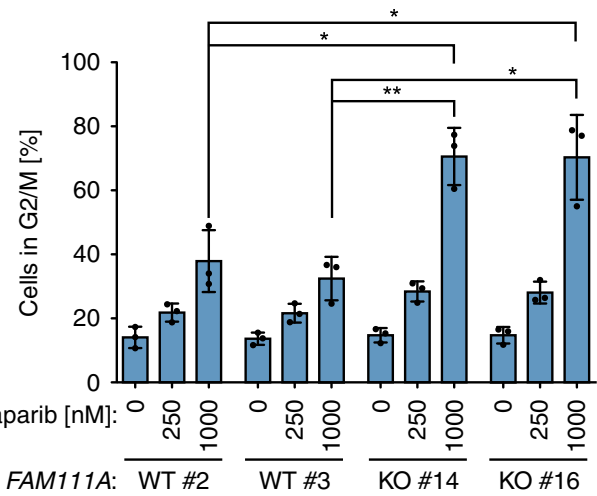

d

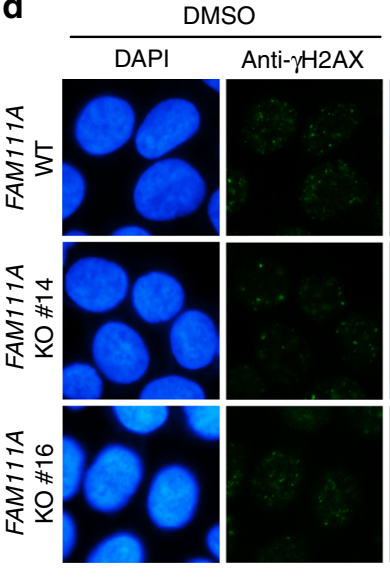

Niraparib

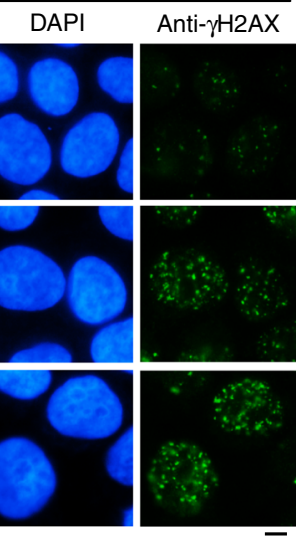

e
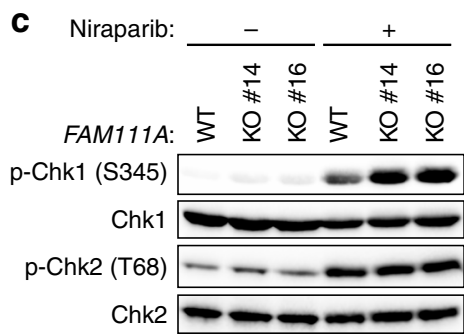

f

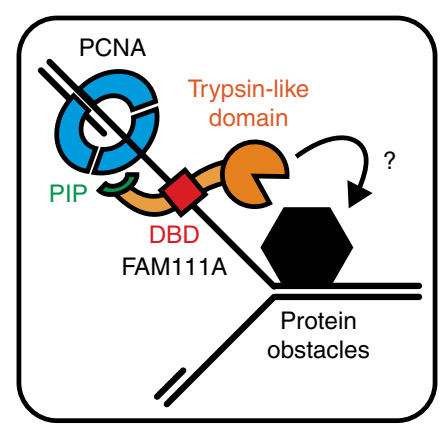

Fig. 6 FAM111A deficiency causes replication stress, DNA damage and cell-cycle arrest during PARPi treatment. a Cell-cycle profiling. Diploid HAP1 clones (WT \#2 and \#3) and FAM111A KO HAP1 clones (\#14 and \#16) were treated with indicated concentration of niraparib or DMSO for $24 \mathrm{~h}$ and analyzed by flow cytometry. Representative histograms from three independent experiments are shown. b Quantification of cells in G2/M phase. Experiments were performed as in a. Percentages of cells in G2/M phase were quantitated using ModFit. Values are mean $\pm \mathrm{s}$.d. of independent experiments $(n=3) .{ }^{\star} p<0.05,{ }^{\star \star} p<0.01$ (two-tailed unpaired $t$-test). $\mathbf{c}$ Levels of $p$-Chk1 and $p$-Chk2 after niraparib treatment. Indicated HAP1 cells were treated with $1.5 \mu \mathrm{M}$ niraparib or DMSO for $24 \mathrm{~h}$ and indicated proteins were detected by Western blotting. $\mathbf{d} \gamma \mathrm{H} 2 \mathrm{AX}$ foci after niraparib treatment. Parental HAP1 (WT) or FAM111A KO clones (\#14 and \#16) were treated with $250 \mathrm{nM}$ niraparib or DMSO for $24 \mathrm{~h}$ and stained with anti- $\gamma \mathrm{H} 2 \mathrm{AX}$ antibody and DAPI. Scale bar, $5 \mu \mathrm{m}$. e Quantification of $\gamma \mathrm{H} 2 \mathrm{AX}$ foci per cell. Experiments were performed as in $\mathbf{d} . \gamma \mathrm{H} 2 \mathrm{AX}$ foci were counted and the distributions of number of foci per cell are plotted (red line: median). ${ }^{\star \star \star} p<0.0001$ (two-tailed unpaired $t$-test). $n$ is the number of individual measures. $\mathbf{f}$ Schematic representation of the working model depicting the role of FAM111A at replication forks encountering protein obstacles. PIP PCNA-interacting peptide box, DBD DNAbinding domain. Source data are provided as a Source Data file.

$4 \mathrm{~h}$ at $4{ }^{\circ} \mathrm{C}$ and the beads were washed five times with NP-40 lysis buffer. Precipitated proteins were analyzed by Western blotting.

Generation of knockout cell lines. CRISPR/Cas9-based gene knockout was performed using pX335 (kind gift from Feng Zhang, Addgene \#42335) for FAM111A and PARP1, and eSpCas9(1.1) (kind gift from Feng Zhang, Addgene \#71814) for SPRTN. For CRISPR/Cas9 using the nickase Cas9 (pX335), the following pairs of gRNAs were used: FAM111A, set 1 (gRNA1 and gRNA2) and set 2 (gRNA3 and gRNA4); PARP1, gRNA1, and gRNA2. The target sequences of gRNA are as follows: FAM111A gRNA1, 5'-ATCAACCCACTAAGTGGCAC-3'; FAM111A gRNA2, 5'-CATATTATTGGCCATCCATAA-3'; FAM111A gRNA3, 5'-ATTATG 
GTTGCCCACTTACT-3'; FAM111A gRNA4, 5'-CAATGTGTAAGGGTGACAT T-3'; PARP1 gRNA1, 5'-CCACCTCAACGTCAGGGTGC-3'; PARP1 gRNA2, 5'-TGGGTTCTCTGAGCTTCGGT-3'; SPRTN gRNA5, 5'-AAGGGGTTCGCTG AGACGGA-3'. Indel mutations were analyzed by Surveyor assays (Surveyor Mutation Detection Kit, Integrated DNA Technologies) and Sanger sequencing of PCR fragments cloned using TOPO TA Cloning (Thermo Fisher). Sequencing results for cell clones used in this study are summarized in Supplementary Fig. 1 and Supplementary Table 1. All HAP1 clones were diploid based on FACS analyses. DNA oligos for cloning and PCR amplification of target regions are summarized in Supplementary Table 3.

Clonogenic survival assays. Cells were seeded in triplicate in six-well plates, allowed to attach on plates for $4 \mathrm{~h}$, and treated with various agents. For CPT (Sigma), niraparib (Merck), etoposide (Sigma), cisplatin (Teva Generics), and 5-aza-2'-deoxycytidine (Sigma), drug containing media were replenished every other day. For talazoparib (Selleckchem), media were not changed during treatment. For formaldehyde (Sigma), cells were treated for the first $24 \mathrm{~h}$ and the medium was changed. Cells were cultured for 6 days, fixed, and stained with Coomassie Blue. Colonies containing more than 50 cells were counted manually.

Flow cytometry. For detection of cell death, cells were stained first with Annexin V-APC (BD Bioscience, 550474) in binding buffer (10 mM HEPES-NaOH pH 7.4, $140 \mathrm{mM} \mathrm{NaCl}, 2.5 \mathrm{mM} \mathrm{CaCl}_{2}$ ) for $15 \mathrm{~min}$ at room temperature, and then with $100 \mathrm{\mu g} \mathrm{ml}^{-1}$ of PI in binding buffer. For analyses of DNA content, cells were fixed with $70 \% \mathrm{EtOH}$ and stained with PI solution $\left(50 \mu \mathrm{g} \mathrm{m}{ }^{-1} \mathrm{PI}, 10 \mu \mathrm{g} \mathrm{ml}^{-1}\right.$ RNase A, $0.05 \%$ NP-40). Two diploid clones of HAP1 (WT \#2 and WT \#3) were used as controls for cell-cycle analyses. Stained cells were analyzed by BD FACS Canto II (BD Biosiences). Percentages of cells in G2/M phase were estimated using ModFit LT (Verity Software House).

Immunofluorescence and microscopy. For $\gamma \mathrm{H} 2 \mathrm{AX}$ and RAD51 immunofluorescence, cells were fixed with $4 \%$ paraformaldehyde for $10 \mathrm{~min}$, permeabilized with $0.2 \%$ Triton X-100 in PBS for $10 \mathrm{~min}$, and blocked with $1 \%$ normal goat serum (for $\gamma \mathrm{H} 2 \mathrm{AX}$ ) or 3\% BSA (for RAD51) in PBS for $1 \mathrm{~h}$. For TOP1cc staining, cells were fixed with $4 \%$ paraformaldehyde for $15 \mathrm{~min}$ at $4{ }^{\circ} \mathrm{C}$, permeabilized with $0.25 \%$ Triton X-100 in PBS for 15 min at $4{ }^{\circ} \mathrm{C}$ and treated with $1 \%$ SDS in PBS for $5 \mathrm{~min}$ at room temperature. Cells were washed five times with wash buffer $(0.1 \%$ Triton X-100, 0.1\% BSA in PBS) and blocked with $10 \%$ milk in $10 \mathrm{mM}$ Tris-HCl pH 7.4 and $150 \mathrm{mM} \mathrm{NaCl}$. Primary antibodies used were mouse anti-phosphoHistone H2A.X (Ser139) (Millipore, 05-636, 1:500), rabbit anti-RAD51 (Calbiochem, PC130, 1:300), and mouse anti-TOP1cc antibody ${ }^{53}$ (1:100). Secondary antibodies used were goat anti-mouse IgG Alexa fluor 488 (Invitrogen, A11029, 1:2000) and goat anti-rabbit IgG Alexa fluor 488 (Invitrogen, A11034, 1:2000). Images were captured using a Zeiss Axio Scope.A1 fluorescent microscope equipped with a CCD camera (Jenoptik). Foci were scored using ImageJ on images captured with a $63 \times$ objective (for $\gamma \mathrm{H} 2 \mathrm{AX}$ ) or counted manually (for RAD51 and TOP1cc). At least 100 cells were scored for focus formation by blinded observers.

DNA combing assay. Cells were labeled with $100 \mu \mathrm{M}$ CldU (Sigma) for $30 \mathrm{~min}$, washed three times with pre-warmed PBS, and labeled with $100 \mu \mathrm{M}$ IdU (Sigma) for an additional $30 \mathrm{~min}$. After labeling cells with IdU, cells were immediately washed three times with ice-cold PBS to inhibit DNA replication. For studying DNA replication under genotoxic stress, the IdU labeling was performed in the presence of a drug ( $3 \mu \mathrm{M}$ Niraparib or $30 \mathrm{nM} \mathrm{CPT})$. To measure collapsed forks, cells were treated with $5 \mathrm{mM} \mathrm{HU}$ for $30 \mathrm{~min}$ after CldU labeling $(30 \mathrm{~min})$ and released in IdU-containing media for $60 \mathrm{~min}$. Where indicated, $5 \mu \mathrm{M}$ ATR inhibitor (VE-821) was added during CldU labeling and HU treatment. DNA fibers were extracted in agarose plugs with FiberPrep DNA Extraction kit (Genomic Vision) and stretched onto silanized coverslips using the FiberComb Molecular Combing System (Genomic Vision). Combed DNA was dehydrated in an oven at $65^{\circ} \mathrm{C}$ for $4 \mathrm{~h}$ and denatured with $0.5 \mathrm{M} \mathrm{NaOH}$ and $1 \mathrm{M} \mathrm{NaCl}$ for 8 min. Samples were then washed three times with PBS for 5 min each on a shaker, dehydrated sequentially in 70,90 , and $100 \%$ ethanol for 5 min each and dried at room temperature for $10 \mathrm{~min}$. Samples were blocked with Block Aid (Invitrogen, B10710) at $37^{\circ} \mathrm{C}$ for 30 min and incubated with rat anti-CldU antibody (Abcam, ab6326, 1:25) and anti-IdU antibody (BD Biosciences, 347580, 1:5) in Block Aid at $37^{\circ} \mathrm{C}$ for $1 \mathrm{hr}$. After washing with PBS-T $(0.05 \%$ Tween-20), samples were incubated with goat anti-rat IgG Alexa 488 (Invitrogen, A11006, 1:200) and goat anti-mouse IgG Alexa 594 (Invitrogen, A11032, 1:200) in Block Aid at $37^{\circ} \mathrm{C}$ for 30 min. After washing with PBS-T, samples were mounted with ProLong Gold (Invitrogen). DNA fibers were photographed on a Zeiss Axio Scope.A1 fluorescent microscope and the length of CldU and IdU tracts were measured by blinded observers using ImageJ.

Purification of recombinant proteins. Recombinant protein production in insect cells was performed essentially as described before ${ }^{19}$ with modifications. pDEST20/ FAM111A-strep, pFastBac NT-B/FAM111A-strep and pFastBac NT-B/StrepFAM111A were transformed into the DH10Bac E. coli. Purified bacmids were transfected into Sf21 in using Cellfectin II (Thermo Fisher Scientific) as instructed by the manufacturer. After incubation at $28^{\circ} \mathrm{C}$ for 3 days, virus-containing supernatants (P1 virus) were transferred to suspension culture in Sf-900 III SFM (Thermo Fisher Scientific) and viruses were amplified for 3 days at $28^{\circ} \mathrm{C}$ ( $\mathrm{P} 2$ virus). Viral titer was measured by baculoQUANT (Oxford Expression Technologies) as instructed. If necessary, the $\mathrm{P} 2$ virus was further amplified (P3 virus). For protein production, Sf21 cells in Sf-900 III SFM supplemented with glucose, yeastolate, and lactalbumin were infected with virus stocks at M.O.I of 10-20 and cultured for 3 days at $28^{\circ} \mathrm{C}$. These cells were collected, washed once with PBS, frozen in liquid $\mathrm{N}_{2}$, and stored at $-80^{\circ} \mathrm{C}$ until protein purification. For protein purification, cell pellets were thawed and resuspended in lysis buffer (50 mM HEPES- $\mathrm{NaOH}$ pH 7.5, $1 \mathrm{M} \mathrm{NaCl}, 1 \mathrm{mM} \mathrm{MgCl}_{2}, 10 \%$ glycerol, $1 \% \mathrm{NP}-40$ ) supplemented with $4 \mathrm{U} \mathrm{ml}^{-1}$ Benzonase (Novagen). For GST-FAM111A-strep, $1 \mathrm{mM}$ PMSF and cOmplete Mini EDTA-free Protease Inhibitor Cocktail (Roche) were added to the lysis buffer. For FAM111A-strep and Strep-FAM111A, $1 \mathrm{mM}$ DTT, $1 \mathrm{mM}$ EDTA and $2 \mu \mathrm{M}$ Pepstatin A were added to the lysis buffer. After 30-min incubation on ice, samples were sonicated and cell debris was removed by centrifugation $\left(20,000 \times g, 4^{\circ} \mathrm{C}\right.$, $30 \mathrm{~min}$ ). Strep-Tactin superflow resin equilibrated with lysis buffer was mixed with lysates by rotation at $4{ }^{\circ} \mathrm{C}$ for $2-3 \mathrm{~h}$. Resins were washed twice with lysis buffer and once with wash buffer (50 mM HEPES- $\mathrm{NaOH}$ pH 7.5, $250 \mathrm{mM} \mathrm{NaCl}, 10 \%$ glycerol). For FAM111A-strep and Strep-FAM111A, $1 \mathrm{mM}$ DTT was included in the wash buffer. Proteins were eluted with elution buffer (wash buffer with $10 \mathrm{mM} \mathrm{d}$ Desthiobiotin), frozen in liquid $\mathrm{N}_{2}$, and stored at $-80^{\circ} \mathrm{C}$.

For protein production in $E$. coli, pMALII-c2x vectors were transformed into BL21 (DE3). For MBP-FAM111A WT 1-99, 100-175, 176-282 and 283-611, cells were cultured until $\mathrm{OD}_{600}=0.5-1.2$ and protein expression was induced with $0.1 \mathrm{mM}$ IPTG at $18^{\circ} \mathrm{C}$ overnight. Other MBP-FAM111A proteins were produced at the basal level expression without IPTG at $37^{\circ} \mathrm{C}$ overnight. Cells were collected, washed with PBS, frozen in liquid $\mathrm{N}_{2}$, and stored at $-80^{\circ} \mathrm{C}$ until protein purification. For protein purification, frozen cells were thawed and resuspended in PBS with $1 \mathrm{mM}$ PMSF and $250 \mathrm{\mu g} \mathrm{ml}^{-1}$ lysozyme. After $30 \mathrm{~min}$ incubation on ice, cells were sonicated and cell debris was removed by centrifugation $\left(20,000 \times g, 4^{\circ} \mathrm{C}\right.$, $30 \mathrm{~min}$ ). Amylose resin (New England Biolab) equilibrated with PBS was mixed with lysates at $4{ }^{\circ} \mathrm{C}$ for $2-3 \mathrm{~h}$. Resins were washed with MBP wash buffer $(50 \mathrm{mM}$ Tris- $\mathrm{HCl} \mathrm{pH} \mathrm{8.0,500} \mathrm{mM} \mathrm{NaCl,} 10 \%$ glycerol, $0.1 \% \mathrm{NP}-40$ ) three times and proteins were eluted with MBP elution buffer (50 mM Tris- $\mathrm{HCl} \mathrm{pH} 8.0,100 \mathrm{mM}$ $\mathrm{NaCl}, 10 \%$ glycerol, $10 \mathrm{mM}$ maltose). Purified proteins were frozen in liquid $\mathrm{N}_{2}$ and stored at $-80^{\circ} \mathrm{C}$.

Electrophoretic mobility shift assay (EMSA). EMSA was performed essentially as described before ${ }^{54}$ with modification. 5' IRDye700-labeled 75-nt DNA oligo (O3063) was synthesized by IDT. Double-stranded (ds), Y-fork, and ds Y-fork DNA substrates were generated by annealing labeled $\mathrm{O} 3063$ with other non-labeled DNA oligos (ds: O4786; Y-fork: O4787; ds Y-fork: O4787 + O3730 + O3757) in annealing buffer ( $10 \mathrm{mM}$ Tris- $\mathrm{HCl} \mathrm{pH} \mathrm{8.0,50} \mathrm{mM} \mathrm{NaCl,} 1 \mathrm{mM}$ EDTA). DNA oligo sequences are shown in Supplementary Table 3. DNA oligos were separated on 6\% non-denaturing polyacrylamide gels and the properly annealed DNA was extracted in $\mathrm{ddH}_{2} \mathrm{O}$ overnight at room temperature. Remaining gels were removed and the buffer was exchanged to TE by Micro Bio-spin six column (Bio-Rad). For EMSA, serially diluted recombinant FAM111A proteins were mixed with 0.7 pmol of DNA substrates in binding buffer (final $25 \mathrm{mM}$ Tris- $\mathrm{HCl} \mathrm{pH} 7.5,5 \mathrm{mM} \mathrm{MgCl}, 10 \%$ glycerol, $20 \mathrm{ng} \mathrm{hl}^{-1} \mathrm{BSA}, 1 \mathrm{mM}$ DTT, total $10 \mu \mathrm{l}$ ) and incubated on ice for $1 \mathrm{~h}$. For experiments using MBP-FAM111A 1-282, protein purity was assessed and protein amounts that contain roughly equal levels of full-length proteins were used. Samples were run on $6 \%$ non-denaturing polyacrylamide gel using $0.5 \times \mathrm{TBE}\left(4^{\circ} \mathrm{C}\right.$, 100-120 V, 90-100 min) and imaged with Odyssey (LI-COR) or ChemiDoc MP (Bio-Rad). Images were analyzed using Image Studio Lite (LI-COR) or Image Lab (Bio-Rad) and percentages of band shifts were calculated based on the band intensities of free and shifted DNA substrates.

Fluorescence polarization DNA-binding assay. DNA binding was measured by fluorescence polarization assays essentially as described befor ${ }^{20}$ with modifications. Serially diluted FAM111A protein (Strep-FAM111A or MBP-FAM111A 176-282) or control protein (BSA or MBP) was mixed with 5' 6-FAM-labeled 31nt DNA oligos (IDT) (final $10 \mathrm{nM}$ ) in FP buffer ( $10 \mathrm{mM}$ HEPES-NaOH pH 7.5, $150 \mathrm{mM} \mathrm{NaCl}$, total $30 \mu \mathrm{l})$. DNA oligo sequences are from previous study $(\mathrm{O} 1676)^{54}$ (Supplementary Table 3). Samples were transferred to black 384-well plate and fluorescence polarization was measured on a Cytation 1 imager (BioTek). Kinetic constants were calculated by four-parameter logistic curve fitting in Prism 5 (GraphPad).

Peptide sequencing. Recombinant FAM111A-strep R569H protein was produced in Sf21 cells and strep-tag containing protein fragments were captured by StrepTactin Super Flow Plus as described above. The sample was separated by SDSPAGE and transferred to a PVDF membrane. Proteins were stained with Coomassie Brilliant Blue R-250 and the cleaved C-terminal fragment was sequenced by Edman degradation peptide sequencing (five cycles) at Alphalyse Laboratories.

Substrate docking analyses. Homology modeling of the FAM111A trypsin-like domain (residues 343-587) was performed using SWISS-MODEL ${ }^{55}$. The homology 
model was constructed using as a template the crystal structure of the plant protease Deg1 in active conformation (Protein Data Bank ID: 3QO6 chain A) ${ }^{42}$, possessing $23.7 \%$ amino acid identity to the modeled region of FAM111A. The substrate peptide sequences were initially positioned in the active site by backbone alignment with corresponding residues of a peptide substrate present in the Deg1 crystal structure.

In preparation for docking, the structural models were imported using ProteinPreparation-Wizard with Maestro 2019 version 19-3 (Schrödinger, LLC) with the OPLS3 force field. Bond orders were assigned, zero-order bonds to metals were determined, disulfide bonds were created, and all hydrogens were generated. Hydrogen bond assignments were applied based on water sampling, and protonation states were predicted for $\mathrm{pH} 7.4 \pm 1.0$ using PROPKA ${ }^{56,57}$. Steric clashes were resolved using a convergence RMSD of $0.3 \AA$ using the OPLS 3 force field under Polak-Ribiere Conjugate Gradient (PRCG) minimization ${ }^{58}$ for 5000 steps, or until the energy difference between subsequent structures was $<0.001 \mathrm{~kJ}$ mol- $\AA^{-156}$.

For molecular docking of peptide substrate sequences, substrates were docked into the binding site using Glide extra precision (XP) peptide-docking protocols (Glide, 19-3, Schrödinger, LLC), which allowed for multiple molecular conformations to be sampled ${ }^{59-62}$. The starting conformations based on the PRCG minimized state were further energy minimized with a water-based solvent to generate charges at an extended cutoff (VdW at $8.0 \AA$, electrostatic at $15 \AA$, H-bond at $4.0 \AA$ ). Soft restraints were placed on all residues $>12 \AA$ from the modeled substrate by using harmonic restraints of $100 \mathrm{kcal} \mathrm{mol}^{-1}$, thus allowing the residues within the cutoff to freely refine position. In addition, the Monte Carlo Molecular Dynamics (MCMD) module with Maestro was employed for sampling side chain conformations and refining atomic positions of substrate-contacting residues within the FAM111A trypsin-like domain to optimize fitting with the substrate, as previously described ${ }^{62-67}$. Docking scores were used to rank the three sequences docked, namely, RTTFGKV, RTTRGKV, and RTTGGKV ${ }^{60-62}$.

Sequence analyses. The degree of amino acid conservation among species was analyzed by the ConSurf server ${ }^{68}$.

Cell fractionation. Cell fractionation was performed as described previously ${ }^{9}$. Briefly, trypsinized cells were washed with PBS and resuspended in hypotonic buffer (100 mM MES-NaOH pH 6.4, 1 mM EDTA, $0.5 \mathrm{mM} \mathrm{MgCl}_{2}$ ) supplemented with protease inhibitor cocktail. After mixing by gentle pipetting, samples were layered on hypotonic buffer containing $30 \%$ sucrose and centrifuged for $10 \mathrm{~min}$ at $15,000 \times g$ at $4^{\circ} \mathrm{C}$. The pellet was isolated and proteins were extracted sequentially by resuspending the pellet in buffer A ( $50 \mathrm{mM}$ HEPES-NaOH pH 7.5, $100 \mathrm{mM}$ $\mathrm{KCl}, 2.5 \mathrm{mM} \mathrm{MgCl}_{2}, 0.05 \%$ Triton X-100, protease inhibitor cocktail), buffer B (50 mM HEPES- $\mathrm{NaOH}$ pH 7.5, $250 \mathrm{mM} \mathrm{KCl,} 2.5 \mathrm{mM} \mathrm{MgCl}_{2}, 0.05 \%$ Triton X-100, protease inhibitor cocktail) and buffer C (50 mM HEPES-NaOH pH 7.5, $500 \mathrm{mM}$ $\mathrm{KCl}, 2.5 \mathrm{mM} \mathrm{MgCl}, 0.1 \%$ Triton X-100, protease inhibitor cocktail). In each step, pellet was isolated by centrifugation $\left(15,000 \times g, 4^{\circ} \mathrm{C}, 10 \mathrm{~min}\right)$. After these extractions, chromatin proteins were released by micrococcal nuclease (NEB) in buffer A supplemented with $5 \mathrm{mM} \mathrm{CaCl} 2$ at $37^{\circ} \mathrm{C}$ for $15 \mathrm{~min}$. Samples were centrifuged $\left(15,000 \times g, 4^{\circ} \mathrm{C}, 10 \mathrm{~min}\right)$ and released proteins in supernatant were analyzed by Western blotting.

iPOND. 3xFlag-FAM111A (WT, S541A or F231A) were stably expressed in $293 \mathrm{~T}$ cells by lentiviral vectors and iPOND experiments were performed essentially as described previously ${ }^{69}$. Briefly, cells were labeled with $10 \mu \mathrm{M}$ EdU for $20 \mathrm{~min}$, and chased with $10 \mu \mathrm{M}$ thymidine for $30 \mathrm{~min}$. Harvested cells were fixed with $1 \%$ formaldehyde for $20 \mathrm{~min}$, permeabilized by $0.25 \%$ Triton X-100 for $30 \mathrm{~min}$ at room temperature followed by $90 \mathrm{~min}$ of click reaction, in which biotin is conjugated to EdU in the EdU-labeled DNA. Cells were lysed in RIPA lysis buffer (50 mM Tris$\mathrm{HCl} \mathrm{pH} 7.4,150 \mathrm{mM} \mathrm{NaCl}, 1 \mathrm{mM}$ EDTA, $1 \%$ NP-40, 0.1\% SDS, 0.5\% Sodium deoxycholate) supplemented with protease inhibitor cocktail and PMSF and sonicated. Precipitates were removed by centrifugation $\left(15,000 \times g, 15 \mathrm{~min}, 4^{\circ} \mathrm{C}\right)$ and EdU-labeled DNA-protein complexes were pulled down by streptavidin agarose beads (EMD Millipore) at $4^{\circ} \mathrm{C}$ overnight. Beads were washed once with RIPA lysis buffer, once with $1 \mathrm{M} \mathrm{NaCl}$, once with PBS, and twice with RIPA lysis buffer. Pulled down proteins were eluted in 2xLithium dodecyl sulfate (LDS) sample buffer at $70^{\circ} \mathrm{C}$ for $1 \mathrm{~h}$ and analyzed by Western blotting.

Statistics and reproducibility. GraphPad Prism 5 was used to graph the data and statistical significance was determined by a two-tailed unpaired $t$-test. Experiments were repeated at least twice and similar results were obtained.

Reporting summary. Further information on research design is available in the Nature Research Reporting Summary linked to this article.

\section{Data availability}

The source data underlying Figs. 1b, d, 2a, c, e, f, 4b, c, f, 5b-e, g, i, 6b, e, and Supplementary Figs. 2b-d, f, 3f, i, k, 1, 5c, 6c, and e are provided as a Source Data file. The uncropped blots underlying Figs. 1a, 2a, b, e, f, 3a-c, e, g-i, 4a, d, 5f, h, 6c, and
Supplementary Figs. 2a, 3a-c, e, f, h-k, 4a, c, 5a, 6a, and d are provided in Supplementary Fig. 7. All data are available from the authors upon reasonable request.

Received: 28 August 2019; Accepted: 24 February 2020; Published online: 12 March 2020

\section{References}

1. Zeman, M. K. \& Cimprich, K. A. Causes and consequences of replication stress. Nat. Cell Biol. 16, 2-9 (2014).

2. Cortez, D. Preventing replication fork collapse to maintain genome integrity. DNA Repair (Amst.) 32, 149-157 (2015).

3. Saldivar, J. C., Cortez, D. \& Cimprich, K. A. The essential kinase ATR: ensuring faithful duplication of a challenging genome. Nat. Rev. Mol. Cell Biol. 18, 622-636 (2017)

4. Ide, H., Nakano, T., Shoulkamy, M. I. \& Salem, A. M. H. Formation, repair, and biological effects of DNA-protein cross-link damage. In Advances in DNA Repair (ed. Chen, C.) 43-80 (IntechOpen, 2015).

5. Tretyakova, N. Y., Groehler, At \& Ji, S. DNA-protein cross-links: formation, structural identities, and biological outcomes. Acc. Chem. Res. 48, 1631-1644 (2015).

6. Ide, H., Nakano, T., Salem, A. M. H. \& Shoulkamy, M. I. DNA-protein crosslinks: formidable challenges to maintaining genome integrity. DNA Repair (Amst.) 71, 190-197 (2018)

7. Pommier, Y. Topoisomerase I inhibitors: camptothecins and beyond. Nat. Rev. Cancer 6, 789-802 (2006).

8. Satoh, M. S. \& Lindahl, T. Role of poly(ADP-ribose) formation in DNA repair Nature 356, 356-358 (1992).

9. Murai, J. et al. Trapping of PARP1 and PARP2 by Clinical PARP Inhibitors Cancer Res. 72, 5588-5599 (2012).

10. Murai, J. et al. Stereospecific PARP trapping by BMN 673 and comparison with olaparib and rucaparib. Mol. Cancer Ther. 13, 433-443 (2014).

11. Shen, Y., Aoyagi-Scharber, M. \& Wang, B. Trapping Poly(ADP-Ribose) polymerase. J. Pharmacol. Exp. Ther. 353, 446-457 (2015).

12. Pommier, Y., O'Connor, M. J. \& de Bono, J. Laying a trap to kill cancer cells: PARP inhibitors and their mechanisms of action. Sci. Transl. Med. 8, 362ps17 (2016).

13. Murai, J. \& Pommier, Y. PARP trapping beyond homologous recombination and platinum sensitivity in cancers. Annu. Rev. Cancer Biol. 3, 131-150 (2019).

14. Bryant, H. E. et al. Specific killing of BRCA2-deficient tumours with inhibitors of poly(ADP-ribose) polymerase. Nature 434, 913-917 (2005).

15. Farmer, H. et al. Targeting the DNA repair defect in BRCA mutant cells as a therapeutic strategy. Nature 434, 917-921 (2005).

16. Stingele, J., Schwarz, M. S., Bloemeke, N., Wolf, P. G. \& Jentsch, S. A DNA dependent protease involved in DNA-protein crosslink repair. Cell 158, 327-338 (2014)

17. Stingele, J. \& Jentsch, S. DNA-protein crosslink repair. Nat. Rev. Mol. Cell Biol. 16, 455-460 (2015)

18. Stingele, J., Habermann, B. \& Jentsch, S. DNA-protein crosslink repair: proteases as DNA repair enzymes. Trends Biochem. Sci. 40, 67-71 (2015)

19. Stingele, J. et al. Mechanism and regulation of DNA-protein crosslink repair by the DNA-dependent metalloprotease SPRTN. Mol. Cell 64, 688-703 (2016).

20. Vaz, B. et al. Metalloprotease SPRTN/DVC1 orchestrates replication-coupled DNA-protein crosslink repair. Mol. Cell 64, 704-719 (2016).

21. Lopez-Mosqueda, J. et al. SPRTN is a mammalian DNA-binding metalloprotease that resolves DNA-protein crosslinks. Elife 5, e21491 (2016)

22. Stingele, J., Bellelli, R. \& Boulton, S. J. Mechanisms of DNA-protein crosslink repair. Nat. Rev. Mol. Cell Biol. 18, 563-573 (2017).

23. Vaz, B., Popovic, M. \& Ramadan, K. DNA-protein crosslink proteolysis repair Trends Biochem. Sci. 42, 483-495 (2017).

24. Morocz, M. et al. DNA-dependent protease activity of human Spartan facilitates replication of DNA-protein crosslink-containing DNA. Nucleic Acids Res. 45, 3172-3188 (2017).

25. Fielden, J., Ruggiano, A., Popovic, M. \& Ramadan, K. DNA protein crosslink proteolysis repair: from yeast to premature ageing and cancer in humans. DNA Repair (Amst.) 71, 198-204 (2018)

26. Li, F., Raczynska, J. E., Chen, Z. \& Yu, H. Structural insight into DNAdependent activation of human metalloprotease Spartan. Cell Rep. 26, 3336-3346 e4 (2019).

27. Maskey, R. S. et al. Spartan deficiency causes accumulation of Topoisomerase 1 cleavage complexes and tumorigenesis. Nucleic Acids Res. 45, 4564-4576 (2017).

28. Duxin, J. P., Dewar, J. M., Yardimci, H. \& Walter, J. C. Repair of a DNAprotein crosslink by replication-coupled proteolysis. Cell 159, 346-357 (2014). 
29. Larsen, N. B. et al. Replication-coupled DNA-protein crosslink repair by SPRTN and the proteasome in xenopus egg extracts. Mol. Cell 73, 574-588 e7 (2019).

30. Ruijs, M. W. et al. Atypical progeroid syndrome: an unknown helicase gene defect? Am. J. Med. Genet. A 116A, 295-299 (2003).

31. Lessel, D. et al. Mutations in SPRTN cause early onset hepatocellular carcinoma, genomic instability and progeroid features. Nat. Genet. 46, 1239-1244 (2014)

32. Maskey, R. S. et al. Spartan deficiency causes genomic instability and progeroid phenotypes. Nat. Commun. 5, 5744 (2014).

33. Borgermann, N. et al. SUMOylation promotes protective responses to DNAprotein crosslinks. EMBO J. 38, e101496 (2019).

34. Alabert, C. et al. Nascent chromatin capture proteomics determines chromatin dynamics during DNA replication and identifies unknown fork components. Nat. Cell Biol. 16, 281-293 (2014).

35. Bochkarev, A., Pfuetzner, R. A., Edwards, A. M. \& Frappier, L. Structure of the single-stranded-DNA-binding domain of replication protein A bound to DNA. Nature 385, 176-181 (1997).

36. Hedstrom, L. Serine protease mechanism and specificity. Chem. Rev. 102, 4501-4524 (2002).

37. Rawlings, N. D. et al. The MEROPS database of proteolytic enzymes, their substrates and inhibitors in 2017 and a comparison with peptidases in the PANTHER database. Nucleic Acids Res. 46, D624-D632 (2018).

38. Unger, S. et al. FAM111A mutations result in hypoparathyroidism and impaired skeletal development. Am. J. Hum. Genet. 92, 990-995 (2013).

39. Isojima, T. et al. A recurrent de novo FAM111A mutation causes KennyCaffey syndrome type 2. J. Bone Miner. Res. 29, 992-998 (2014)

40. Nikkel, S. M. et al. Mother-to-daughter transmission of Kenny-Caffey syndrome associated with the recurrent, dominant FAM111A mutation $\mathrm{p}$. Arg569His. Clin. Genet. 86, 394-395 (2014).

41. Abraham, M. B. et al. Short stature and hypoparathyroidism in a child with Kenny-Caffey syndrome type 2 due to a novel mutation in FAM111A gene. Int. J. Pediatr. Endocrinol. 2017, 1 (2017).

42. Kley, J. et al. Structural adaptation of the plant protease Deg1 to repair photosystem II during light exposure. Nat. Struct. Mol. Biol. 18, 728-731 (2011).

43. Prasad, R. et al. Suicidal cross-linking of PARP-1 to AP site intermediates in cells undergoing base excision repair. Nucleic Acids Res. 42, 6337-6351 (2014).

44. Prasad, R., Horton, J. K., Dai, D. P. \& Wilson, S. H. Repair pathway for PARP1 DNA-protein crosslinks. DNA Repair (Amst.) 73, 71-77 (2019).

45. Sparks, J. L. et al. The CMG helicase bypasses DNA-protein cross-links to facilitate their repair. Cell 176, 167-181 e21 (2019).

46. Clausen, T., Southan, C. \& Ehrmann, M. The HtrA family of proteases: implications for protein composition and cell fate. Mol. Cell 10, 443-455 (2002).

47. Clausen, T., Kaiser, M., Huber, R. \& Ehrmann, M. HTRA proteases: regulated proteolysis in protein quality control. Nat. Rev. Mol. Cell Biol. 12, 152-162 (2011).

48. Krojer, T. et al. Structural basis for the regulated protease and chaperone function of DegP. Nature 453, 885-890 (2008).

49. Fine, D. A. et al. Identification of FAM111A as an SV40 host range restriction and adenovirus helper factor. PLoS Pathog. 8, e1002949 (2012).

50. Panda, D., Fernandez, D. J., Lal, M., Buehler, E. \& Moss, B. Triad of human cellular proteins, IRF2, FAM111A, and RFC3, restrict replication of orthopoxvirus SPI-1 host-range mutants. Proc. Natl Acad. Sci. USA 114, 3720-3725 (2017).

51. Tarnita, R. M., Wilkie, A. R. \& DeCaprio, J. A. Contribution of DNA replication to the FAM111A-mediated simian virus 40 host range phenotype. J. Virol. 93, e01330 (2019).

52. Machida, Y., Kim, M. S. \& Machida, Y. J. Spartan/C1orf124 is important to prevent UV-induced mutagenesis. Cell Cycle 11, 3395-3402 (2012).

53. Patel, A. G. et al. Immunodetection of human topoisomerase I-DNA covalent complexes. Nucleic Acids Res. 44, 2816-2826 (2016).

54. Toth, A., Hegedus, L., Juhasz, S., Haracska, L. \& Burkovics, P. The DNAbinding box of human SPARTAN contributes to the targeting of Poleta to DNA damage sites. DNA Repair (Amst.) 49, 33-42 (2017).

55. Waterhouse, A. et al. SWISS-MODEL: homology modelling of protein structures and complexes. Nucleic Acids Res. 46, W296-W303 (2018).

56. Sondergaard, C. R., Olsson, M. H., Rostkowski, M. \& Jensen, J. H. Improved treatment of ligands and coupling effects in empirical calculation and rationalization of pKa values. J. Chem. Theory Comput. 7, 2284-2295 (2011).

57. Li, H., Robertson, A. D. \& Jensen, J. H. Very fast empirical prediction and rationalization of protein pKa values. Proteins 61, 704-721 (2005).

58. Polak, E. \& Ribiere, G. Note sur la convergence de méthodes de directions conjuguées. Esaim Math. Model Numer Anal 3, 35-43 (1969).

59. Caulfield, T. \& Devkota, B. Motion of transfer RNA from the A/T state into the A-site using docking and simulations. Proteins 80, 2489-2500 (2012).

60. Loving, K., Salam, N. K. \& Sherman, W. Energetic analysis of fragment docking and application to structure-based pharmacophore hypothesis generation. J. Comput. Aided Mol. Des. 23, 541-554 (2009).
61. Salam, N. K., Nuti, R. \& Sherman, W. Novel method for generating structurebased pharmacophores using energetic analysis. J. Chem. Inf. Model. 49, 2356-2368 (2009)

62. Caulfield, T. \& Medina-Franco, J. L. Molecular dynamics simulations of human DNA methyltransferase 3B with selective inhibitor nanaomycin A. J. Struct. Biol. 176, 185-191 (2011).

63. Caulfield, T. R. et al. Phosphorylation by PINK1 releases the UBL domain and initializes the conformational opening of the E3 ubiquitin ligase Parkin. PLoS Comput. Biol. 10, e1003935 (2014).

64. Abdul-Hay, S. O. et al. Selective targeting of extracellular insulin-degrading enzyme by quasi-irreversible thiol-modifying inhibitors. ACS Chem. Biol. 10, 2716-2724 (2015)

65. Batra, J. et al. Long-range electrostatic complementarity governs substrate recognition by human chymotrypsin $\mathrm{C}$, a key regulator of digestive enzyme activation. J. Biol. Chem. 288, 9848-9859 (2013).

66. Lopez-Vallejo, F. et al. Integrating virtual screening and combinatorial chemistry for accelerated drug discovery. Comb. Chem. High. Throughput Screen. 14, 475-487 (2011).

67. Paulus, A. et al. Coinhibition of the deubiquitinating enzymes, USP14 and UCHL5, with VLX1570 is lethal to ibrutinib- or bortezomib-resistant Waldenstrom macroglobulinemia tumor cells. Blood Cancer J. 6, e492 (2016)

68. Ashkenazy, H. et al. ConSurf 2016: an improved methodology to estimate and visualize evolutionary conservation in macromolecules. Nucleic Acids Res. 44 W344-W350 (2016).

69. Sirbu, B. M. et al. Analysis of protein dynamics at active, stalled, and collapsed replication forks. Genes Dev. 25, 1320-1327 (2011).

\section{Acknowledgements}

We thank Larry M. Karnitz for critical reading of the manuscript, Kevin L. Peterson for technical assistance, and Karen S. Flatten for technical advice. This work was supported by the National Institutes of Health (R01 CA233700 to Y.J.M.) and Eagles Cancer Research Fund to Y.J.M.

\section{Author contributions}

Conceptualization, Y.J.M., Y.K., and S.H.K.; Investigation, Y.K., Y.M., Y.J.M., and S.P.; Formal analysis, T.R.C. and E.S.R.; Writing-Original Draft, Y.K. and Y.J.M.; Writing-Review \& Editing, Y.M.J., Y.K., S.H.K., T.R.C. and E.S.R.; Funding Acquisition, Y.J.M.; Resources, S.H.K.; Supervision, Y.J.M.

\section{Competing interests}

S.H. Kaufmann is an inventor on U.S. patent No. 8,530,172 held by Mayo Clinic that deals with use of antibodies to protein-DNA complexes as diagnostic reagents to predict responsiveness to topoisomerase inhibitors. The remaining authors declare no competing interests.

\section{Additional information}

Supplementary information is available for this paper at https://doi.org/10.1038/s41467 020-15170-7.

Correspondence and requests for materials should be addressed to Y.J.M.

Peer review information Nature Communications thanks the anonymous reviewer(s) for their contribution to the peer review of this work. Peer reviewer reports are available.

Reprints and permission information is available at http://www.nature.com/reprints

Publisher's note Springer Nature remains neutral with regard to jurisdictional claims in published maps and institutional affiliations.

pen Access This article is licensed under a Creative Commons Attribution 4.0 International License, which permits use, sharing, adaptation, distribution and reproduction in any medium or format, as long as you give appropriate credit to the original author(s) and the source, provide a link to the Creative Commons license, and indicate if changes were made. The images or other third party material in this article are included in the article's Creative Commons license, unless indicated otherwise in a credit line to the material. If material is not included in the article's Creative Commons license and your intended use is not permitted by statutory regulation or exceeds the permitted use, you will need to obtain permission directly from the copyright holder. To view a copy of this license, visit http://creativecommons.org/ licenses/by/4.0/.

(C) The Author(s) 2020 